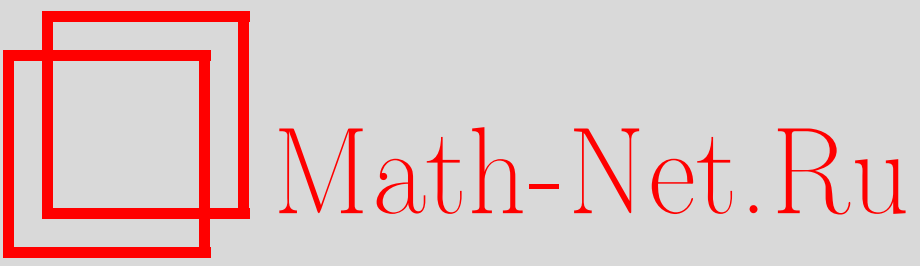

В. А. Артамонов, Общие квантовые многочлены: неприводимые модули и Морита-эквивалентность, Изв. РАН. Сер. матем., 1999, том 63, выпуск 5, 3-36

DOI: https://doi.org/10.4213/im259

Использование Общероссийского математического портала Math-Net.Ru подразумевает, что вы прочитали и согласны с пользовательским соглашением

http://www.mathnet.ru/rus/agreement

Параметры загрузки:

IP: 54.81 .137 .203

26 апреля 2023 г., 14:26:00 
В. А. Артамонов

\title{
Общие квантовые многочлены: неприводимые модули и Морита-эквивалентность
}

\begin{abstract}
В настоящей работе продолжены исследования строения конечно порожденных модулей над кольцами общих квантовых (лорановских) многочленов. Получено описание решетки подмодулей периодического конечно порожденного модуля, описаны неприводимые модули. Исследован вопрос о Морита-эквивалентности колец общих квантовых полиномов, рассматриваются свойства тел частных и решается проблема Зарисского для квантовых многочленов.

Библиографияя: 18 наименований.
\end{abstract}

\section{$\S 1$. Введение}

Настояшая работа продолжает исследования строения конечно порожденных модулей над кольцами $A$ общих квантовых (лорановских) многочленов. Напомним необходимые основные определения и полученные ранее результаты. Пусть $D$ - тело с фиксированными автоморфизмами $\alpha_{1}, \ldots, \alpha_{n}$, где $n \geqslant 2$. Предположим, что выделены элементы $q_{i j} \in D^{*}, i, j=1, \ldots, n$, причем

$$
q_{i i}=q_{i j} q_{j i}=Q_{i j r} Q_{j r i} Q_{r i j}=1, \quad \alpha_{i}\left(\alpha_{j}(d)\right)=q_{i j} \alpha_{j}\left(\alpha_{i}(d)\right) q_{j i},
$$

где $Q_{i j r}=q_{i j} \alpha_{j}\left(q_{i r}\right), d \in D$. Положим

$$
Q=\left(q_{i j}\right) \in \operatorname{Mat}(n, D) \text { и } \alpha=\left(\alpha_{1}, \ldots, \alpha_{n}\right) .
$$

Элементы матрицы $Q$ называются системой мультипараметров. Через

$$
\Lambda=D_{Q, \alpha}\left[X_{1}^{ \pm 1}, \ldots, X_{r}^{ \pm 1}, X_{r+1}, \ldots, X_{n}\right]
$$

обозначим ассоциативное кольцо, порожденное элементами тела $D$, элементами $X_{1}, \ldots, X_{n}, X_{1}^{-1}, \ldots, X_{r}^{-1}$ и заданное определяющими соотношениями

$$
\begin{aligned}
X_{i} X_{i}^{-1} & =X_{i}^{-1} X_{i}=1, & & 1 \leqslant i \leqslant r ; \\
X_{i} d & =\alpha_{i}(d) X_{i}, & & d \in D, \quad i=1, \ldots, n ; \\
X_{i} X_{j} & =q_{i j} X_{j} X_{i}, & & i, j=1, \ldots, n .
\end{aligned}
$$

Это кольцо называется кольцом квантовых полиномов. Как отмечено в [1], кольцо $\Lambda$ из (2) разлагается в скрешенное произведение $\Lambda=D \sharp_{t} H$, где биалгебра $H$ является тензорным произведением целочисленного группового кольца свободной абелевой

Работа частично поддержана РФФИ (гранты № 99-01-00382, № 96-15-96050), программой “Университеты России" (грант № 5527), программой INTAS93-436. 
группы с базой $\left\{X_{i} \mid 1 \leqslant i \leqslant r\right\}$ и целочисленного полугруппового кольца свободной абелевой полугруппы с базой $\left\{X_{i} \mid r<i \leqslant n\right\}$.

Кольцо $\Lambda$ из (2) мы будем также обозначать через

$$
\Lambda=D_{*, *}\left[X_{1}^{ \pm 1}, \ldots, X_{r}^{ \pm 1}, X_{r+1}, \ldots, X_{n}\right]
$$

в случае, когда значения $Q, \alpha$ понимаются однозначно.

Квантовые многочлены тесно связаны с групповыми кольцами бесконечных разрешимых групп (см. ниже). Кроме того, квантовые многочлены возникают внекоммутативной геометрии как алгебры функций на квантовом аффинном пространстве [2], [3, гл. 2, $\S 2.6]$. Квантовые многочлены связаны также с мультипараметрическими квантовыми алгебрами Вейля в смысле [4] (см. также [5], [3]). Именно, как показано в [5], тело частных квантовых алгебр Вейля совпадает с телом частных квантовых многочленов. Отметим в связи с этим работу [6] по телам частных квантовых многочленов.

Кольцо $\Lambda$ из (2) является левым и правым векторным пространством над $D$, базу которого составляют одночлены

$$
u=X_{1}^{m_{1}} \ldots X_{n}^{m_{n}}
$$

где $m_{i} \in \mathbb{Z}$ при $1 \leqslant i \leqslant r$ и $m_{i} \in \mathbb{N} \cup 0$, если $r<i \leqslant n$. В частности, кольцо $\Lambda$ из (2) является нётеровой слева и справа областью с телом частных

$$
F=D_{Q, \alpha}\left(X_{1}, \ldots, X_{n}\right)=D_{*, *}\left(X_{1}, \ldots, X_{n}\right) .
$$

Если $M-$ конечно порожденньй $\Lambda$-модуль, то рангом $M$ называется число

$$
d=\operatorname{dim}_{F} F \otimes_{\Lambda} M .
$$

Каждый автоморфизм $\alpha_{i}$ продолжается на $F$, причем

$$
\alpha_{i}(f)=X_{i} f X_{i}^{-1}
$$

для всех $f \in F$. Каждое подкольцо $L$ в $\Lambda$ из (2), порожденное $D$ и частью неизвестных, а также, возможно, их обратными, инвариантно относительно указанных продолжений автоморфизмов $\alpha_{1}, \ldots, \alpha_{n}$. Пусть $S$ - множество всех ненулевых элементов из $L$. Тогда $S^{-1} L$ является телом частных в $L$, в котором продолжают действовать автоморфизмы $\alpha_{1}, \ldots, \alpha_{n}$ из (6). Таким образом, можно построить кольцо квантовых многочленов над телом $S^{-1} L$ от оставшихся переменных $X_{j}$ и, возможно, их обратных, если $j \leqslant r$. Снова получается кольцо квантовых многочленов, содержащее $\Lambda$ в качестве подкольца. Эти соображения показывают, что $S$ удовлетворяют условию Оре в $\Lambda$.

В [7] показано, что если $D=k$ - поле, $\alpha$ - набор тождественных автоморфизмов $k$, TO

$$
k_{Q, \alpha}\left[X_{1}^{ \pm 1}, \ldots, X_{n}^{ \pm 1}\right] \simeq k_{Q^{\prime}, \alpha}\left[Y_{1}^{ \pm 1}, \ldots, Y_{n}^{ \pm 1}\right]
$$

тогда и только тогда, когда существует такая матрица

$$
M=\left(m_{i j}\right) \in \mathrm{GL}(n, \mathbb{Z}),
$$

что

$$
q_{i j}^{\prime}=\prod_{r, s} q_{r s}^{m_{r i} m_{s j}}
$$


ОПРЕДЕЛЕНИЕ 1.1. Пусть $N$ - подгруппа в мультипликативной группе $D^{*}$, порожденная коммутантом $\left[D^{*}, D^{*}\right]$ и всеми элементами вида $z^{-1} \alpha_{i}(z)$, где $z \in D^{*}$ и $i=$ $1, \ldots, n$.

Заметим, что $N$ является нормальной подгруппой в мультипликативной группе $D^{*}$, причем $\bar{D}=D^{*} / N$ является мультипликативной абелевой группой.

В работах [1] - [10] предполагается, что $D$ удовлетворяет условию

(*) образы всех әлементов $q_{i j}, \quad 1 \leqslant i<j \leqslant n$, независимы в $\bar{D}$.

Это условие впервые рассмотрено в [7]. В этом случае будем говорить, что кольца

$$
A=D_{Q, \alpha}\left[X_{1}^{ \pm 1}, \ldots, X_{n}^{ \pm 1}\right], \quad C=D_{Q, \alpha}\left[X_{1}, \ldots, X_{n}\right]
$$

являются соответственно кольцом общих квантовых лорановских многочленов и кольцом общих квантовых многочленов. Кольцо $\Lambda$ мы будем также называть кольцом общих квантовых многочленов. Отметим, что свойство кольца быть общим кольцом сохраняется при переходе к подкольцу, порожденному телом $D$ и частью переменных, а также при локализациях по всем ненулевым элементам из указанного подкольца.

Укажем пример колец общих квантовых многочленов. Пусть группа $W$ порождена элементами

$$
X=\left\{X_{i} \mid 1 \leqslant i \leqslant n\right\}
$$

Предположим, что $W$ обладает нормальным рядом

$$
W=W_{0}>W_{1}>W_{2}>\ldots>W_{t+1}=1
$$

с конечно порожденными свободными абелевыми факторами $W_{i} / W_{i+1}$, причем базу $W / W_{1}$ составляют элементы множества

$$
Y=\left\{X_{i} W_{1} \mid 1 \leqslant i \leqslant n, \quad X_{i} \in X\right\}
$$

а базу $W_{1} / W_{2}$ составляют элементы

$$
\left\{q_{i j} W_{2} \mid 1 \leqslant i<j \leqslant n, \quad q_{i j}=\left[X_{i}, X_{j}\right]\right\}
$$

Предположим, что в качестве $\alpha_{1}, \ldots, \alpha_{n}$ взяты внутренние автоморфизмы группы $W$, соответствуюшие сопряжениям с помошњю $X_{1}, \ldots, X_{n}$. В частности,

$$
\alpha_{i}\left(q_{i j}\right) \equiv q_{i j} \quad\left(\bmod W_{1}\right)
$$

Пусть $k$ - некоторое поле. По $[11, \S 11]$ мультипликативная полугруппа $S=k W_{1} \backslash$ 0 удовлетворяет условию Оре в групповой алгебре $k W$, и поэтому сушествует тело частных $D$ групповой алгебры $k W_{1}$. Тогда в качестве кольца $A$ из $(7)$ можно брать кольцо $S^{-1} k W$.

Напомним ряд полученных ранее результатов. Следующая теорема доказана сначала в частном случае в [7], а затем в обшем случае в [1] (см. [8, теорема 3.13$])$. 
Теорема 1.2. Пусть $A-$ кольцо общих квантовых лорановских многочленов из (7). Тогда K. $\operatorname{dim} A=1$. Другими словами, пусть $1 \leqslant i_{1}<\ldots<$ $i_{h} \leqslant n-$ набор индексов, где $h \leqslant n-2, u 1 \leqslant j_{1}<\ldots<j_{n-h} \leqslant n-$ все оставииеся индексы от 1 до п. Положим

$$
G=D_{*, *}\left(X_{i_{1}}, \ldots, X_{i_{h}}\right)
$$

Если $M$ - конечномерное (левое) векторное пространство над $G$, являющееся модулем над $G\left[X_{j_{1}}^{ \pm 1}, \ldots, X_{j_{n-h}}^{ \pm 1}\right]$, то $M=0$.

Теорема 1.3 [1]. Если выполнено условие (*), то все конечно порожсденные проективнье модули над кольцом $\Lambda$ из (2) ранга не меньше двух свободны.

Напомним, что элемент $m$ модуля $M$ над кольцом $\Lambda$ из (2) называется периодичес$\kappa и м$, если $a m=0$ для некоторого $a \in \Lambda \backslash 0$. Через $t(M)$ обозначается периодическая часть модуля $M$, т.е. множество всех его периодических элементов. Так как кольцо $\Lambda$ из (2) является нётеровой слева и справа областью, то оно удовлетворяет условию Оре. Следовательно, периодическая часть $t(M)$ модуля $M$ является подмодулем в $M$.

Теорема 1.4 [9]. Пусть выполнено условие (*), кольцо А из (7) и $M$ - конечно порожденный $A$-модуль ранга $r \geqslant 0$. Тогда $M=t(M) \oplus N$, где $t(M)$ - чиклический артинов $A$-модуль, $N$ - проективный $A$-модуль ранга $r$. Eсли $r=0$, то $N=0$. Если $r=1$, то модуль $N$ изоморфен левому идеалу в $A$. Если $r>1$, то модуль $N$ свободен.

Ситуация, в некотором смысле противоположная условию (*), рассмотрена в [12], [13]. Например, пусть выполнено следуюшее условие:

(**): $\alpha_{1}, \ldots, \alpha_{n}-$ тождественные автоморфизмы, тело $D$ конечномерно над $k$, и все мультипараметры $q_{i j}$ являются корнями из 1.

Теорема 1.5 [12]. Пусть выполнено условие (**) и задан конечно порожденный проективный $\Lambda$-модуль $Р$ ранга не меньше двух. Тогда модуль $Р$ свободен.

Теорема 1.6 [13]. Пусть выполнено условие (**). Eсли $m \geqslant 4$ и $U \in \operatorname{GL}(m, \Lambda)$, mo

$$
U=\operatorname{diag}(z u, 1, \ldots, 1) U_{1},
$$

где $и$-обратимый одночлен из $\Lambda, z \in D^{*} u U_{1} \in E(m, \Lambda)$.

Целью настояшей работы является описание решетки подмодулей периодических (циклических) модулей, в частности, строения неприводимых модулей над кольцом обших квантовых (лорановских) многочленов (см. теорему 2.16), решение вопроса о Морита-эквивалентности обших квантовых многочленов (теоремы $6.16,6.18$ ) и решение проблемы Зарисского для обших квантовых многочленов (см. теоремы 7.1, 7.2).

В связи с рассмотрением вопроса о Морита-эквивалентности колец квантовых многочленов отметим книгу [14], а также близкие работы [17], [15], [16]. Отметим, что подробный обзор полученных результатов по проективным модулям над квантовыми многочленами можно найти в [8]. 
Всюду в работе все модули предполагаются левыми. Для любого кольца $K$ через $\mathbb{P}(K)$ обозначается категория конечно порожденных проективных левых $K$-модулей. Через $B$ обозначается подкольцо

$$
B=D_{*, *}\left[X_{1}^{ \pm 1}, \ldots, X_{n-1}^{ \pm 1}\right]
$$

в $A$. Заметим, что $A=B\left[X_{n}^{ \pm 1}, \alpha_{n}\right]-$ кольцо косых многочленов с коэффициентами в $B$.

Все необходимые определения и полезные результаты относительно нётеровых некоммутативных колец можно найти в [14].

\section{§ 2. Неприводимые модули}

Элемент

$$
f \in A=B\left[X_{n}^{ \pm 1}, \alpha_{n}\right], \quad f=\sum_{i=d}^{q} b_{i} X_{n}^{i}, \quad b_{i} \in B,
$$

называется унитарныцм по $X_{n}$, если коэффишиенты $b_{d}, b_{q}$ обратимы в $B$, т.е. $b_{d}, b_{q}$ являются одночленами от $X_{1}, \ldots, X_{n-1}$. Несложно доказывается

Предложение 2.1 [14, предложение 9.3]. Элемент $f \in A$ является унитарным по $X_{n}$ тогда и только тогда, когда $A / A f$ является конечно порохсденным $B$-модулем.

Предложение 2.2 [14, предложение 9.3]. Пусть $U=U(A)$ - множсество всех унитарных по $X_{n}$ әлементов из $A$. Тогда $U$ удовлетворяет левому условию Оре.

В дальнейшем нам потребуется

Предложение 2.3. Пусть в кольце А из (7) задан ненулевой әлемент

$$
f=\sum_{i_{1}, \ldots, i_{n} \in \mathbb{Z}} a_{i_{1}, \ldots, i_{n}} X_{1}^{i_{1}} \ldots X_{n}^{i_{n}}, \quad a_{i_{1}, \ldots, i_{n}} \in D .
$$

Предположим, что $p>2 \max _{j=1, \ldots, n}\left|i_{j}\right|$. Положим

$$
Y_{j}=\left\{\begin{array}{lll}
X_{j} X_{n}^{p^{j}}, & \text { eсли } & j<n \\
X_{n}, & \text { если } & j=n
\end{array}\right.
$$

Тогда

$$
A=D_{*, *}\left[Y_{1}^{ \pm 1}, \ldots, Y_{n}^{ \pm 1}\right]
$$

причем $f$ как многочлен относительно $Y_{1}, \ldots, Y_{n}$ унитарен по $Y_{n}$.

ДоКАЗАТЕЛЬСТво.Для каждого одночлена из (9) имеем

$$
a_{i_{1}, \ldots, i_{n}} X_{1}^{i_{1}} \ldots X_{n}^{i_{n}}=a_{i_{1}, \ldots, i_{n}}^{\prime} Y_{1}^{i_{1}} \ldots Y_{n-1}^{i_{n-1}} Y_{n}^{i_{n}+p i_{1}+\ldots+p^{n-1} i_{n-1}} .
$$

Следовательно, разные одночлены из (9) имеют разные показатели при $Y_{n}$. Таким образом, коэффициенты при наибольшей и наименьшей степенях $Y_{n}$ являются одночленами относительно $Y_{1}, \ldots, Y_{n-1}$.

Отметим, что при указанных заменах свойство кольца $A$ быть общим кольцом не меняется [10]. 
Предложение 2.4. Пусть задан $A$-модуль $M$, являющийся конечно породсденным модулем над подкольцом

$$
D_{*, *}\left[X_{1}^{ \pm 1}, \ldots, X_{n-2}^{ \pm 1}\right]
$$

Тогда $M=0$.

ДокАЗАТЕЛЬСтво. Пусть $M \neq 0$ и $S$ - множество ненулевых элементов из (10). Тогда $S$ удовлетворяет условию Оре в $A$ и $S^{-1} M$ является модулем над

$$
S^{-1} A=D_{*, *}\left(X_{1}, \ldots, X_{n-2}\right)\left[X_{n-1}^{ \pm 1}, X_{n}^{ \pm 1}\right],
$$

имеющим конечную размерность над телом частных (10). Следовательно, $S^{-1} M=0$ по теореме 1.2. Таким образом, $M$ является периодическим конечно порожденным модулем над (10). По теореме 1.4 модуль $M$ цикличен над (10) с порождающим элементом $m$. Пусть $f$ - ненулевой элемент из кольца (10), аннулируюший $m$. По предложению 2.3 , совершая замену переменных $X_{1}, \ldots, X_{n-2}$, можно считать, что $f$ унитарен по $X_{n-2}$. Тогда модуль $M$ конечно порожден над

$$
D_{*, *}\left[X_{1}^{ \pm 1}, \ldots, X_{n-3}^{ \pm 1}\right] .
$$

Продолжая этот процесс, получим, что $M$ имеет конечную размерность над $D$, что снова противоречит теореме 1.2 .

Предложение 2.5. Пусть задан $A$-модуль $M$, являющийся $B$-модулем с образующими $m_{1}, \ldots, m_{n}$, причем существуют такие әлементы $b_{1}, \ldots, b_{n} \in B \backslash 0$, что $b_{i} m_{i}=0$ для всех $i=1, \ldots, n$. Тогда $M=0$.

ДокАЗАТЕльство. Так как $B \backslash 0$ удовлетворяет условию Оре в $A$, то существует такой элемент $b \in B \backslash 0$, что $b m_{1}=\ldots=b m_{n}=0$. Как и в [9], совершая в $B$ замену переменных вида

$$
Y_{i}= \begin{cases}X_{i} X_{n-1}^{p^{i}}, & \text { если } i<n-1, \\ X_{i}, & \text { если } i=n-1,\end{cases}
$$

для достаточно большого натурального числа $p$, можно считать, что элемент $b \in B$ унитарен по $X_{n-1}$. По предложению $2.1 M$ является конечно порожденным модулем над

$$
D_{*, *}\left[Y_{1}^{ \pm 1}, \ldots, Y_{n-2}^{ \pm 1}\right] .
$$

Остается воспользоваться предложением 2.4 .

Следствие 2.6. Пусть задан $A$-модуль $M$, являющийся конечно порожденным В-модулем. Тогда периодическая часть Т в $М$ равна нулю.

ДоказАтельство. Так как $B \backslash 0$ удовлетворяет условию Оре, то $T$ является $A$-подмодулем в $M$, причем $T$ - конечно порожденньй $B$-модуль, ибо $B$ нётерово слева. Остается применить к $T$ предложение 2.5.

Следствие 2.7. Пусть задан А-модуль $M$, являющийся конечно порожденным.м В-модулем. Тогда $M \in \mathbb{P}(B)$. 
ДокАЗАТЕЛЬСтво. Нужно воспользоваться следствием 2.6 и [9, теорема 1.6] (см.теорему 1.4).

Пусть $H$ - тело частных кольца $B$. Как отмечено в (6), автоморфизм $\alpha_{n}$ продолжается на $H$. Так как $B \backslash 0$ удовлетворяет условию Оре в $A$, то

$$
R=(B \backslash 0)^{-1} A=H\left[X_{n}^{ \pm 1}, \alpha_{n}\right] .
$$

Всюду в дальнейшем через $R$ будем обозначать кольцо из (11).

Если $M$ - модуль над произвольным кольцом $K$, то через $L_{K}(M)$ обозначается решетка $K$-подмодулей в $M$.

Предложение 2.8. Пусть $M$ - конечно порожденный периодический $A$-модуль. Совериая замену переменных вида (11), как и выше, можно считать, что $M \in \mathbb{P}(B)$. Имеется естественный изоморфизм решеток подмодулей

$$
\varphi: L_{A}(M) \rightarrow L_{R}\left(R \otimes_{A} M\right)
$$

индуиированный вложением $M$ в $R \otimes_{A} M$.

ДокАЗАТЕльСтво. Заметим, что ядро естественного гомоморфизма

$$
M \rightarrow R \otimes_{A} M
$$

совпадает с подмодулем $T$ из следствия 2.6. Поэтому естественный гомоморфизм из $M$ в $R \otimes_{A} M$ является вложением. Пусть $U \supseteq V$ - подмодули в $M$, причем

$$
R \otimes_{A} U=H \otimes_{B} U=H \otimes_{B} V=R \otimes_{A} V,
$$

и поэтому

$$
R \otimes_{A}(U / V)=H \otimes_{B}(U / V)=0 .
$$

Так как кольцо $A$ нётерово слева и справа, то модуль $U$ конечно порожден. Из (12) получаем, что модуль $U / V$ удовлетворяет условиям предложения 2.5 , и поэтому $U / V=$ 0 , т.е. $U=V$. Отсюда вытекает, что $\varphi$ является вложением.

Обратно, пусть задан подмодуль $L$ в $R \otimes_{A} M$ и $h \in L$. Тогда $b h \in L \cap M$ для некоторого $b \in B \backslash 0$. Следовательно, $h=b^{-1}(b h) \in H \otimes_{B}(L \cap M)$. Таким образом, $L=H \otimes_{B}(L \cap M)$ и $\varphi$ сюръективно.

Следствие 2.9. Для $A$-модуля $M \in \mathbb{P}(B)$ следующие условия әквивалентны:

1) $M$ неприводим;

2) $R$-модуль $R \otimes_{A} M$ неприводим.

Следствие 2.10. Пусть задань $A$-модули $M_{1}, M_{2} \in \mathbb{P}(B) u$

$$
f: R \otimes_{A} M_{1} \rightarrow R \otimes_{A} M_{2}
$$

- гомоморфизм R-модулей. Тогда $f\left(M_{1}\right) \subseteq M_{2}$. Другими словами, $f$ индуцировано гомоморфизмом $A$-модулей $\left.f\right|_{M_{1}}: M_{1} \rightarrow M_{2}$. 
ДоКАЗАТЕЛЬСТВо. Заметим, что $M=f\left(M_{1}\right)+M_{2}$ является конечно порожденным $B$-модулем, причем $R \otimes_{A} M=R \otimes_{A} M_{2}$. По предложению 2.8 получаем $M=M_{2}$. Отсюда $f\left(M_{1}\right) \subseteq M_{2}$.

Следствие 2.11. Если заданы $A$-модули $M_{1}, M_{2} \in \mathbb{P}(B)$, то любой изоморфизм

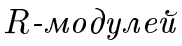

$$
f: R \otimes_{A} M_{1} \rightarrow R \otimes_{A} M_{2}
$$

индуцирован изоморфизмом А-модулей $\left.f\right|_{M_{1}}: M_{1} \rightarrow M_{2}$. В частности, если $R \otimes_{A} M_{1}=R \otimes_{A} M_{2}$, mo $M_{1}=M_{2}$.

Пусть, как и выше, задан $A$-модуль $M \in \mathbb{P}(B)$. Нетрудно видеть (см. теорему 1.4 ), что $A$-модуль $M$ периодичен. Следовательно, по [9, теорема 1.6] модуль $M$ является циклическим, т.е. $M \simeq A / I$ для некоторого левого идеала $I \subseteq A$. Так как $R$ - кольцо главных левых идеалов, то $I=R f \cap A$ для некоторого $f \in R \backslash 0$.

Предложение 2.12. Пусть $f \in R \backslash 0$ и задан изоморфизм левых $А$-модулей $M \simeq A /(R f \cap A)$. Следующие условия әквивалентны:

1) $M \in \mathbb{P}(B)$;

2) $R f \cap A$ содержит унитарный по $X_{n}$ многочлен из $A$.

ДоказАТЕЛЬСТво. Пусть выполнено условие 1) и $M=A /(R f \cap A)$. Положим $m=1+(R f \cap A)$. Так как кольцо $B$ нётерово слева и $M \in \mathbb{P}(B)$, то существует такое натуральное число $r$, что

$$
X_{n}^{r} m, X_{n}^{-1} m \in \sum_{i=0}^{r-1} B X_{n}^{i} m .
$$

В частности, найдутся такие $b_{i}, c_{i} \in B$, что

$$
\left(X_{n}^{r}+\sum_{i=0}^{r-1} b_{i} X_{n}^{i}\right) m=\left(X_{n}^{-1}+\sum_{i=0}^{r-1} c_{i} X_{n}^{i}\right) m=0
$$

Положим

$$
g=X_{n}^{-1}+\sum_{i=0}^{r-1}\left(b_{i}+c_{i}\right) X_{n}^{i}+X_{n}^{r}
$$

Поскольку $g \in A$ унитарен и $g m=0$, то $g \in R f \cap A$. Итак, выполнено условие 2$)$.

Обратно, пусть выполнено условие 2$)$ и $g \in R f \cap A$ - унитарный многочлен. Тогда $A / A g \in \mathbb{P}(B)$ по предложению 2.1. Следовательно, существует эпиморфизм $A$-модулей $A / A g \rightarrow M$. Остается воспользоваться следствием 2.7 .

Используя соображения из предложения 2.12 для $A$-модуля $M \in \mathbb{P}(B)$, можно описать решетку $L(M)=L\left(R \otimes_{A} M\right)$. Имеем $M=A /(R f \cap A)$, причем $R f \cap A$ содержит унитарный по $X_{n}$ элемент. При этом

$$
R \otimes_{A} M=R / R f
$$


Так как $R$ - кольцо главных левых идеалов, то имеется взаимно однозначное соответствие между подмодулями в $R \otimes_{A} M$ и правыми делителями $h$ элемента $f$ в $R$. Именно, если $f=u h$, где $u, h \in R$, то $h$ сопоставляется подмодуль

$$
M(h)=(R h \cap A) /(R f \cap A) .
$$

При этом $M(h) \supseteq M\left(h^{\prime}\right)$ тогда и только тогда, когда $h^{\prime} \in R h$. Заметим, что

$$
H \otimes_{B} M(h) \simeq R h / R f \simeq R / R u
$$

Таким образом, по следствиям 2.9, 2.10 получаем $M(h) \simeq A /(R u \cap A)$.

ОПреДЕЛЕНИЕ 2.13. Пусть $D U$ - множество всех таких $f \in R \backslash 0$, что $R f \cap A$ содержит ненулевой элемент из множества $U(A)$ всех унитарных по $X_{n}$ элементов из $A$. Другими словами, $D U$ состоит из всех таких $f \in R$, что $R f \cap U(A)$ непусто.

Предложение 2.14. DU является мультипликативной полугруппой.

ДокАЗАтЕльство. Пусть $f, g \in D U$. Тогда существуют такие $h_{1}, h_{2} \in R$, что $h_{1} f, h_{2} g \in U(A)$. Достаточно показать, что $R h_{1} f g \cap U(A)$ непусто. Можно считать, что $h_{1}=1, f \in U(A)$. Кроме того, можно предполагать, что $h=h_{2} \in A$. Так как $U(A)$ удовлетворяет условию Оре, то найдутся такие $f_{1} \in U(A), f_{2} \in A$, что $f_{1} f h=f_{2} f$. Но $f_{1} f h g=f_{2} f g$, откуда $f_{1} f(h g) \in U(A) \cap A f g$ и $f g \in D U$.

ОПРЕДЕЛЕНИЕ 2.15. Элемент $f \in A \backslash 0$ (соответственно $f \in R \backslash 0)$ неприводим по $X_{n}$ в $A$ (соответственно в $R$ ), если из условия $f=g h$, где $g, h \in A$, (соответственно $g, h \in R$ ), вытекает, что либо $g$, либо $h$ обратим в $A$ (соответственно в $R$ ).

Из предыдуших рассмотрений получаем, что справедлива

Теорема 2.16. Пусть задан $А$-модуль $M \in \mathbb{P}(B)$. Следующие условия эквивалентни:

1) $A$-модуль $M$ неприводим;

2) $M \simeq A /(R f \cap A)$, где $f \in R \backslash 0$ неприводим $и f \in D U$, т.е. $R f \cap A$ содержит унитарный по $X_{n}$ многочлен.

ДокАЗАТЕЛЬСтво. По предложению 2.12 можно считать, что $M=A /(R f \cap A)$, причем $R f \cap A$ содержит унитарный в $A$ элемент. При этом $R \otimes_{A} M=R / R f$. Остается воспользоваться следствием 2.9 .

Теорема 2.16 имеет следуюшую интерпретацию. Пусть $A$-модуль $M$ неприводим и $M \in \mathbb{P}(B)$. Предположим, что $m \in M \backslash 0$, причем элементы

$$
m, X_{n} m, \ldots, X_{n}^{r-1} m, \quad r \geqslant 1
$$

независимы над $B$, но

$$
b_{r} X_{n}^{r} m=\sum_{i=0}^{r-1} b_{i} X_{n}^{i} m
$$


для некоторых $b_{i} \in B$, причем $b_{r} \neq 0$. Тогда

$$
f=b_{r} X_{n}^{r}-\sum_{i=0}^{r-1} b_{i} X_{n}^{i}
$$

является многочленом из условия теоремы 2.16 , причем $R f \cap A$ содержит унитарный по $X_{n}$ многочлен. Элементы вида (13) образуют базис $H \otimes_{B} M=R \otimes_{A} M$ над телом $H$. В силу теоремы 2.16 указанным способом получается любой неприводимьй $A$-модуль.

Итак, каждый неприводимый $A$-модуль параметризуется натуральным чис-лом $p$, связанным с заменой переменных вида $(11)$, и неприводимым многочленом $f$ из теоремы 2.16.

Предположим, что $A$-модуль $M$ задается одним числом $p$ и двумя неприводимыми многочленами $f_{1}, f_{2} \in R$. Это соответствует тому, что в $M$ выбраны два образующих $m_{1}, m_{2} \in M \backslash 0$, причем $R f_{1}$ и $R f_{2}$ - аннуляторы $m_{1}, m_{2} \in M$. Предположим, что $m_{2}=h m_{1}, h \in R \backslash R f_{1}$. Тогда $f_{2} h \in R f_{1}$, т.е. $f_{2} h=u f_{1}$ для некоторого $u \in R$. Итак,

$$
f_{2}\left(A \backslash R f_{1}\right) \cap R f_{1} \neq 0 .
$$

Обратно, предположим, что $f_{1}, f_{2} \in R$ неприводимы и выполнено (14). Пусть $R f_{1} \cap A$ содержит элемент, унитарный по $X_{n}$. По предположению $f_{2} h=u f_{1}, h \in A \backslash R f_{1}$, $u \in R$. Заметим, что

$$
M \simeq A /\left(A \cap R f_{1}\right) \subseteq R / R f_{1} .
$$

Таким образом, $z=h+R f_{1} \in M \backslash 0$. Так как модуль $M$ неприводим по теореме 2.16 , то $M=A z$. Кроме того, если $w \in R$ и $w z=0$, то $w h \in R f_{1}$.Если бы $w \notin R f_{2}$, то $R w+R f_{2}=R$, поскольку $f_{2}$ неприводим. В этом случае $R h=R w h+R f_{2} h \subseteq R f_{1}$, т.е. $h \in R f_{1}$, что неверно. Итак, $w \in R f_{2}$ и Ann $z=R f_{1}$. По теореме $2.16 R f_{1} \cap A$ содержит элемент, унитарньй по $X_{n}$. Следовательно, если пара $\left(p, f_{1}\right)$ из теоремы 2.16 определяет неприводимый $A$-модуль, то любая пара $\left(p, f_{2}\right)$, где $f_{2}$ - неприводимый элемент из $R$, удовлетворяющий (14), определяет тот же модуль $M$ и наоборот.

\section{§ 3. Примеры}

Рассмотрим ряд примеров неприводимых $A$-модулей.

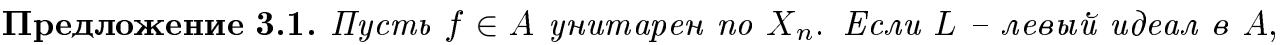
содержащий $A f$, причем $L \cap B \neq 0$, то $L=A$.

ДокАЗАТЕЛЬство. Пусть $M=A / L$. Тогда $M$ является конечно порожденным $A$-модулем. Более того, если $b \in L \cap B$ и $m_{1}=1+L$, то $b m_{1}=0$. Так как $M=A m_{1}$, то остается воспользоваться предложением 2.5.

Предложение 3.2. Пусть $f \in A \backslash 0$ унитарен по $X_{n}$ и неприводим в $R$. Если $w \in A \backslash A f$, mo $A w+A f=A$.

ДокАЗАТЕЛЬСТво. Кольцо $R$ является кольцом главных левых идеалов. Следовательно, по условию $R w+R f=R$. Таким образом, $(A w+A f) \cap B \neq 0$. Остается воспользоваться предложением 3.1 . 
Теорема 3.3. Пусть $f \in A$ унитарен по $X_{n}$. Следующие условия эквивалент$H b l$ :

1) $f$ неприводим в $R$;

2) $f$ неприводим в $A$.

ДоКАЗАТЕЛьСтво. Пусть выполнено условие 1) и $f=g h$, где $g, h \in A$. Предположим, например, что $g \in H$. Тогда $g \in H \cap A=B$. Сравнивая старшие коэффициенты при $X_{n}$ в $f$ и в $g h$, получаем, что $g$ имеет правый обратный в $A$. Но тогда $g$ - одночлен, т.е. $g$ обратим в $A$.

Предположим теперь, что выполнено условие 2$)$ и $f=g h, g, h \in R$. Так как $B \backslash 0$ удовлетворяет условию Оре, то сушествует такой элемент $b \in B \backslash 0$, что $h=b^{-1} u$, где $u \in A$. Заменяя $g$ на $g b^{-1}$ и $h$ на $u$, можно считать, что $h \in A$.

Если $h \in A \backslash A f$, то $A h+A f=A$ по предложению 3.2 . В частности, $1=v h+w f$ для некоторых $v, w \in A$. Но тогда $1=(v+w g) h$, т.е. $h$ обратим в $A$.

Пусть $h \in A f$. Тогда $h=r f$ для некоторого $r \in A \backslash 0$. Отсюда $f=g h=g r f$, т.е. $g$ обратим в $A$.

Теорема 3.4. Пусть $f \in A$ унитарен и неприводим по $X_{n}$ в $A$. Тогда $A$-модуль A/Af неприводим.

ДоКАЗАТЕЛЬСТВо. Нужно воспользоваться предложением 3.2 и теоремой 2.16.

Следуюшее утверждение в качестве следствия приведено в [10]. Изложим его доказательство. Пусть $I$ - левый идеал в $B$. Как показано в [10, предложение 3.3], на $I$ сушествует структура $A$-модуля.

Теорема 3.5. I является неприводимым А-модулем.

ДокАЗАТЕльство. Пусть задан ненулевой $A$-подмодуль $J$ в $I$. Тогда для любого $z \in I$ существует такое $c \in B \backslash 0$, что $c z \in J$. Если $M=I / J$, то по предложению 2.5 получаем $M=0$.

В заключение этого параграфа приведем серию примеров неприводимых $A$-модулей. Для этого в силу теоремы 3.4 укажем серию унитарных неприводимых по $X_{n}$ полиномов из $A$. Пусть $m, n \geqslant 2$ и $f=X_{n}^{m}-X_{n-1}$. Ясно, что $f$ унитарен по $X_{n}$ и по $X_{n-1}$.

Предложение 3.6. Полином $f \in A$ неприводим.

ДоказАтельство. Пусть $f=g h$, где $g, h \in A$. Тогда $g, h$ унитарны по $X_{n}$. Поскольку $f$ унитарен и по $X_{n-1}$, то степени $g, h$ по $X_{n-1}$ равны либо 0 , либо 1 и $g, h$ унитарны по $X_{n-1}$. Пусть, например, степень $g$ по $X_{n-1}$ равна 1 . Можно считать, что $g=a+b X_{n-1}$, где $a, b$ лежат в подкольце $Q$ в $A$, порождаемом $D, X_{1}^{ \pm 1}, \ldots, X_{n-2}^{ \pm 1}, X_{n}^{ \pm 1}$. Кроме того, степень $h$ по $X_{n-1}$ равна нулю, т.е. $h \in Q$. Тогда $a h=X_{n}^{m}, b \alpha_{n-1}(h)=$ -1 . Следовательно, $h$ обратим в $Q$ и поэтому $h$ обратим в $A$. Аналогично рассматривается случай, когда степень $g$ по $X_{n-1}$ равна нулю.

Отметим, что если $f=X_{n}^{m}-X_{n-1}$, то $A / A f$ является свободным $B$-модулем ранга $m$. 


\section{§ 4. Неприводимые модули над многочленами}

Обозначим, как и выше, через $\Lambda$ кольцо (2), где $n>r \geqslant 0$. Опишем неприводимые $\Lambda$-модули.

Предложение 4.1. Пусть задан произвольный $\Lambda$-модуль $M$. Тогда $X_{i} M$ является $\Lambda$-подмодулем в $M$. В частности, если модуль $M$ неприводим, то либо $X_{i} M=0$, либо $X_{i} M=M$.

ДокАЗАТЕЛЬСТво. Нужно воспользоваться соотношением $X_{i} \Lambda=\Lambda X_{i}$.

В силу этого предложения можно считать, что неприводимый $\Lambda$-модуль $M$ является точным. Если $m \in M \backslash 0$, то найдется такое $a \in \Lambda$, что $m=-a X_{r+1} \ldots X_{n} m$, т.е. $\left(1+a X_{r+1} \ldots X_{n}\right) m=0$. В силу [1, предложение 2] модуль $M$ является неприводимым $A$-модулем. Таким образом, в силу теоремы 2.16 получаем $M \simeq$ $\Lambda /(R f \cap \Lambda)$, где $f \in R$ неприводим, $R f \cap \Lambda$ содержит унитарный по $X_{n}$ полином $g$ и, кроме того, $R f \cap \Lambda$ содержит элемент $1+a X_{r+1} \ldots X_{n}$. Отсюда следует, что $R f \cap \Lambda$ содержит элемент

$$
1+a X_{r+1} \ldots X_{n}+\left(X_{r+1} \ldots X_{n}\right)^{d} g
$$

являюшийся унитарным при достаточно большом натуральном числе $d \geqslant 2$.

Теорема 4.2. Пусть задан точный $\Lambda$-модуль $M$, причем $M$ как модуль над $B \cap \Lambda$ конечно порожден. Следующие условия эквивалентны:

1) $M-$ неприводимый $\Lambda$-модуль;

2) $M \simeq \Lambda /(\Lambda \cap R f)$, где $f \in R$ неприводим и в $\Lambda \cap R f$ содержст унитарныц ио $X_{n}$ әлемент $h \equiv 1\left(\bmod X_{r+1} \ldots X_{n} \Lambda\right)$.

ДокАЗАТЕЛЬСтво. Выше показано, что 1) влечет 2). Обратно, пусть выполнено условие 2 ). По [1, предложение 2] модуль $M$ является неприводимым над $A$ и, следовательно, неприводимым $\Lambda$-модулем.

Теорема 4.3. Пусть $f \in \Lambda$ унитарен по $X_{n} u f \equiv 1\left(\bmod X_{r+1} \ldots X_{n} \Lambda\right)$. Элемент $f$ неприводим по $X_{n}$ в $\Lambda$ тогда и только тогда, когда $f$ неприводим по $X_{n}$ в R.

ДокАЗАТЕЛЬСТво. Пусть $f$ неприводим по $X_{n}$ в $R$ и $f=g h$, где $g, h \in \Lambda$, причем, например, степень $g$ по $X_{n}$ меньше степени $f$. Тогда $g \in H \cap \Lambda=B \cap \Lambda$. Сравнивая свободные члены $f$ и $g h$, получаем, что $g$ обратим слева в $B \cap \Lambda$, т.е. $g \in D^{*}$.

Обратно, пусть $f$ неприводим по $X_{n}$ в $\Lambda$ и $f=g h$ в $R$. По теореме 3.3 можно считать, что $g, h \in A$. Кроме того, можно предполагать, что $g=u^{-1} g_{1}, h=v^{-1} h_{1}$, где $u, v-$ одночлены от $X_{r+1}^{-1}, \ldots, X_{n}^{-1}$, и $g_{1}, h_{1} \in \Lambda$, причем их свободные члены отличны от нуля. Отсюда $v u f=\left(v g_{1} v^{-1}\right) h_{1}$. Сравнивая свободные члены, получаем, что $v u=1$, $v g_{1} v^{-1}, h_{1} \in \Lambda$ и $f=\left(v g_{1} v^{-1}\right) h_{1}$ - нетривиальное разложение для $f$ в $\Lambda$.

Следствие 4.4. Пусть $f \in \Lambda$ унитарен по $X_{n} u f \equiv 1\left(\bmod X_{r+1} \ldots X_{n} \Lambda\right)$, причем $f$ неприводим по $X_{n}$ в $\Lambda$. Тогда $\Lambda / \Lambda f-$ неприводимый $\Lambda$-модуль. 
ДОКАЗАТЕЛЬСТво. Нужно воспользоваться теоремами 3.3, 4.2, 4.3.

Укажем серию примеров неприводимых многочленов $f$ в $\Lambda$. Пусть

$$
f=1+X_{r+1} \ldots X_{n}\left(1-X_{n}^{d}\right), \quad n \geqslant 2, \quad d \geqslant 1 .
$$

Этот многочлен неприводим по $X_{n}$ в $\Lambda$. Действительно, пусть $f=g h$, где $g, h \in \Lambda$. Степень $f$ по $X_{1}$ равна 1 . Пусть степень $g$ по $X_{r+1}$ равна нулю. Тогда $h=u+X_{r+1} v$, где $u, v$ лежат в подкольце, порожденном

$$
D, X_{1}^{ \pm 1}, \ldots, X_{r}^{ \pm 1}, X_{r+2}, \ldots, X_{n} .
$$

Отсюда $y u=1$, т.е. $d \in D^{*}$. Аналогично рассматривается случай, когда степень $h$ по $X_{1}$ равна нулю.

\section{§5. Тела частных}

В этом параграфе рассматриваются тела частных произвольных квантовых многочленов. Для любого элемента $f \in C \backslash 0$, где $C$ из (7), через $\bar{f}$ обозначим младший член $f$ относительно лексикографического порядка на одночленах от $X_{1}, \ldots, X_{n}$. Пусть $\bar{f}=$ $\kappa(f) X_{1}^{m_{1}} \ldots X_{n}^{m_{n}}$, где $\kappa(f) \in D^{*}$ и $m_{i} \geqslant 0$. Положим $\pi(f)=\left(m_{1}, \ldots, m_{n}\right) \in \mathbb{Z}^{n}$. Аналогично через $\hat{f}=\tau(f) X_{1}^{s_{1}} \ldots X_{n}^{s_{n}}$ обозначается старший член $f, \rho(f)=\left(s_{1}, \ldots, s_{n}\right) \in$ $\mathbb{Z}^{n}$. Как отмечено в [1], если $f, g \in C \backslash 0$, то

$$
\pi(f g)=\pi(f)+\pi(g), \quad \rho(f g)=\rho(f)+\rho(g)
$$

и по модулю $N$

$$
\kappa(f g)=\kappa(f) \kappa(g) \mu(\pi(f), \pi(g)), \quad \tau(f g)=\tau(f) \tau(g) \mu(\rho(f), \rho(g)),
$$

где для $z=\left(z_{1}, \ldots, z_{n}\right), t=\left(t_{1}, \ldots, t_{n}\right) \in \mathbb{Z}^{n}$

$$
\mu(z, t) \in \prod_{1 \leqslant i<j \leqslant n} q_{i j}^{-z_{j} t_{i}} N \in D^{*} / N .
$$

Тогда отображение $\mu: \mathbb{Z}^{n} \otimes \mathbb{Z}^{n} \rightarrow D^{*} / N$ билинейно. Рассмотрим на множестве $G=$ $D^{*} / N \times \mathbb{Z}^{n}$ операцию

$$
(a, z)(b, t)=(a b \mu(z, t), z+t) .
$$

Несложная проверка показывает, что $G$ относительно этой операции является группой и отображения $\zeta, \psi:(C \backslash 0) \rightarrow G, \zeta(f)=(\kappa(f), \pi(f)), \psi(f)=(\tau(f), \rho(f))$ являются гомоморфизмами полугрупп.

Пусть $z \in F$, где $F$ из (5), и $z=f g^{-1}=h v^{-1}$, где $f, g, h, v \in C \backslash 0$. В силу условия Оре найдутся такие $t, s \in C$, что $g t=v s, f t=h s$. Отсюда

$$
\zeta(f) \zeta(t)=\zeta(h) \zeta(s), \quad \zeta(g) \zeta(t)=\zeta(v) \zeta(s),
$$

и поэтому

$$
\zeta(f) \zeta(g)^{-1}=\zeta(h) \zeta(v)^{-1}
$$


Таким образом, $\zeta$ (и аналогично $\psi$ ) однозначно продолжаются до отображений

$$
\zeta, \psi: F \rightarrow G
$$

Если $f, g, h, v \in C \backslash 0$ и

$$
g t=h s, \quad t, s \in C \backslash 0,
$$

то $f g^{-1} h v^{-1}=f t(v s)^{-1}$. Следовательно,

$$
\begin{aligned}
\zeta\left(f g^{-1} h v^{-1}\right) & =\zeta(f) \zeta(t) \zeta(v)^{-1} \zeta(s)^{-1}=\zeta(f) \zeta(h) \zeta(s) \zeta(g)^{-1} \zeta(v)^{-1} \zeta(s)^{-1} \\
& =\zeta(f) \zeta(g)^{-1} \zeta(h) \zeta(s)^{-1}=\zeta\left(f g^{-1}\right) \zeta\left(h v^{-1}\right) .
\end{aligned}
$$

Итак, $\zeta$ является гомоморфизмом групп. Аналогичное утверждение верно и для $\psi$.

Теорема 5.1. $\left[F^{*}, D^{*}\right] \cap D^{*}=N$. Кроме того, $\left[F^{*}, F^{*}\right] \cap D^{*}-$ nодгруппа в $D^{*}$, порожденная $N$ и всеми $q_{i j}, 1 \leqslant i<j \leqslant n$.

ДокАЗАТЕЛЬСТво. Пусть $z \in D^{*}$ и $1 \leqslant i \leqslant n$. Тогда

$$
z^{-1} \alpha_{i}(z)=z^{-1} X_{i} z X_{i}^{-1} \in\left[F^{*}, D^{*}\right] \cap D^{*}
$$

Отсюда $N \subseteq\left[F^{*}, D^{*}\right] \cap D^{*}$. Кроме того, если $1 \leqslant i, j \leqslant n$, то

$$
q_{i j}=X_{j}^{-1} X_{i}^{-1} X_{j} X_{i} \in\left[F^{*}, F^{*}\right] \cap D^{*}
$$

Обратно, если

$$
z=\prod\left[f_{i}, g_{i}\right] \in D^{*}, \quad f_{i}, g_{i} \in F^{*}
$$

то $\zeta(z)=(z N, 0)$. С другой стороны,

$$
\zeta(z)=\prod\left(\left\{\mu\left(\pi\left(f_{i}\right), \pi\left(g_{i}\right)\right) \mu\left(\pi\left(f_{i} g_{i}\right), \pi\left(f_{i}^{-1} g_{i}^{-1}\right)\right) \mu\left(\pi\left(f_{i}\right)^{-1}, \pi\left(g_{i}\right)^{-1}\right)\right\}, 0\right),
$$

т.е. $z$ лежит в подгруппе, порожденной всеми $q_{i j}$ и $N$. Более того, если $f_{i} \in D^{*}$, то $\zeta(z)=(N, 0)$, и поэтому $z \in N$.

Следствие 5.2. $\Lambda$ является кольцом общих квантовых многочленов тогда и только тогда, когда мультипликативная абелева группа

$$
\left(\left[F^{*}, F^{*}\right] \cap D^{*}\right) /\left(\left[F^{*}, D^{*}\right] \cap D^{*}\right)
$$

имеет максимально возможнный ранг, который равен $n(n-1) / 2$.

Отметим, что группа $D^{*} \times \mathbb{Z}^{r}$ связана с вычислением группь $K_{1}(\Lambda)$ $[18$, c. 122$]$. 
Предложение 5.3. Пусть $C$ - кольцо квантовьх многочленов из (7), и предположим, что

$$
\widehat{C}=\widehat{D}_{\widehat{Q}, \widehat{\alpha}}\left[Y_{1}, \ldots, Y_{m}\right]
$$

- другое кольцо квантовых многочленов, где $\widehat{D}$ - тело с набором автоморфизмов $\widehat{\alpha}=\left(\widehat{\alpha}_{1}, \ldots, \widehat{\alpha}_{m}\right)$ в $\widehat{D}$. Предположим, что задано вложение колеи $\phi: \widehat{C} \rightarrow F$, где $F$ из (5), причем $\phi(\widehat{C}) \phi(\widehat{C} \backslash 0)^{-1}=F, \phi(\widehat{D}) \subseteq D$ и при $i=1, \ldots, m$

$$
\phi\left(Y_{i}\right)=\lambda_{i} X_{1}^{d_{i 1}} \ldots X_{n}^{d_{i n}}, \quad \lambda_{i} \in D^{*}, \quad d_{i j} \in \mathbb{Z} .
$$

Положим

$$
\Delta=\left(d_{i j}\right) \in \operatorname{Mat}(m \times n, \mathbb{Z}) .
$$

Тогда линейное отображсение $\Delta: \mathbb{Z}^{m} \rightarrow \mathbb{Z}^{n}$ сюрвективно $и$, в частности, $m \geqslant n$. Ecлu $m=n$, mo $\Delta \in \mathrm{GL}(n, \mathbb{Z}) u \phi(\widehat{D})=D$.

ДокАЗАТЕЛЬство. По условию сушествуют такие $f, g \in \widehat{C} \backslash 0$, что для $1 \leqslant i \leqslant n$ выполнено равенство $X_{i}=\phi(g) \phi(f)^{-1}$, т.е. $X_{i} \phi(f)=\phi(g)$. Пусть

$$
f=\sum a_{i_{1}, \ldots, i_{m}} Y_{1}^{i_{1}} \ldots Y_{m}^{i_{m}}, \quad g=\sum b_{j_{1}, \ldots, j_{m}} Y_{1}^{j_{1}} \ldots Y_{m}^{j_{m}}
$$

Тогда

$$
\begin{aligned}
& \phi(f)=\sum \phi\left(a_{i_{1}, \ldots, i_{m}}\right) \gamma_{i_{1}, \ldots, i_{m}} \prod_{s=1}^{m}\left(\prod_{j=1}^{n} X_{j}^{d_{s j}}\right)^{i_{s}}, \\
& \phi(g)=\sum \phi\left(b_{j_{1}, \ldots, j_{m}}\right) \gamma_{j_{1}, \ldots, j_{m}} \prod_{s=1}^{m}\left(\prod_{l=1}^{n} X_{l}^{d_{s l}}\right)^{j_{s}},
\end{aligned}
$$

где $\gamma_{j_{1}, \ldots, j_{m}} \in D^{*}$, причем

$$
\gamma_{i_{1}, \ldots, i_{m}} \equiv \prod_{s=1}^{m} \lambda_{s}^{i_{s}} \bmod N
$$

Таким образом, для любого $i=1, \ldots, n$ найдутся такие

$$
\left(i_{1}, \ldots, i_{m}\right),\left(j_{1}, \ldots, j_{m}\right) \in \mathbb{Z}^{m}
$$

что в $\mathbb{Z}^{n}$

$$
\left(i_{1}, \ldots, i_{m}\right) \Delta+(0, \ldots, 0, \stackrel{i}{1}, 0, \ldots, 0)=\left(j_{1}, \ldots, j_{m}\right) \Delta,
$$

где $\Delta$ из (18). Следовательно,

$$
\left(-i_{1}+j_{1}, \ldots,-i_{m}+j_{m}\right) \Delta=(0, \ldots, 0, \stackrel{i}{1}, 0, \ldots, 0)
$$

Таким образом, $\Delta$ задает сюръективное линейное отображение $\Delta: \mathbb{Z}^{m} \rightarrow \mathbb{Z}^{n}$, и поэтомy $m \geqslant n$. 
Пусть $\lambda \in D^{*}$ и $m=n$. По условию найдутся такие $f, g \in \widehat{C}$, что $\lambda=\phi\left(g f^{-1}\right)$, т.е. $\lambda \phi(f)=\phi(g)$. Предположим, что $f, g$ из (19). В силу (20) для любого набора $\left(l_{1}, \ldots, l_{n}\right) \in \mathbb{Z}^{n}$ получаем, что

$$
\sum \lambda \phi\left(a_{i_{1}, \ldots, i_{n}}\right) \gamma_{i_{1}, \ldots, i_{n}}=\sum \phi\left(b_{j_{1}, \ldots, j_{n}}\right) \gamma_{j_{1}, \ldots, j_{n}}
$$

где суммы берутся по всем таким наборам $\left(i_{1}, \ldots, i_{n}\right),\left(j_{1}, \ldots, j_{n}\right) \in \mathbb{Z}^{n}$, что

$$
\sum_{s=1}^{n} i_{s} d_{s j}=\sum_{s=1}^{n} j_{s} d_{s j}=l_{j}, \quad j=1, \ldots, n .
$$

Так как матрица $\Delta$ принадлежит $\mathrm{GL}(n, \mathbb{Z})$, то в $(21)$ с каждой стороны имеется только одно слагаемое, причем $i_{s}=j_{s}$ для всех $s=1, \ldots, n$. Поэтому в (21) получаем

$$
\lambda \phi\left(a_{i_{1}, \ldots, i_{n}}\right)=\phi\left(b_{j_{1}, \ldots, j_{n}}\right),
$$

откуда $\lambda \in \phi(\widehat{D})$.

Предложение 5.4. Пусть $z, w \in F^{*}$, причем $z+w \neq 0$. Тогда $\pi(z+w) \geqslant$ $\min (\pi(z), \pi(w))$, причем равенство имеет место, если $\pi(z) \neq \pi(w)$.

ДокАЗАТЕЛЬСтво. Пусть $z=f g^{-1}, w=h v^{-1}$ и $t, s$ из (16). Тогда

$$
\begin{aligned}
\pi(z+w) & =\pi\left(f g^{-1}+h v^{-1}\right)=\pi(f t+h s)-\pi(v s) \\
& \geqslant \min (\pi(f)+\pi(t), \pi(h)+\pi(s))-\pi(v s) \\
& =\min (\pi(f)-\pi(g), \pi(h)-\pi(v))=\min (\pi(z), \pi(w)) .
\end{aligned}
$$

Если, например, $\pi(z)>\pi(w)$, то $\pi(f t)>\pi(h s)$, и поэтому $\pi(f t+h s)=\pi(f t)$, т.е. $\pi(z+w)=\pi(z)$.

Предложение 5.5. Пусть $z \in F^{*} u \pi(z)=\left(m_{1}, \ldots, m_{n}\right) \in \mathbb{Z}^{n}$. Тогда существует такой элемент $\lambda \in D^{*}$, что либо $z=\lambda X_{1}^{m_{1}} \ldots X_{n}^{m_{n}}$, либо

$$
\pi\left(z-\lambda X_{1}^{m_{1}} \ldots X_{n}^{m_{n}}\right)>\pi(z) .
$$

ДокАЗАТЕЛЬСтво. Пусть $z=u v^{-1}$, где $\bar{u} \bar{v}^{-1}=\lambda X_{1}^{m_{1}} \ldots X_{n}^{m_{n}}$. Тогда $\bar{u}=$ $\lambda X_{1}^{m_{1}} \ldots X_{n}^{m_{n}} \bar{v}$ и $u=\lambda X_{1}^{m_{1}} \ldots X_{n}^{m_{n}} \bar{v}+u^{\prime}$, где $u^{\prime}>\bar{u}$, и $v=\bar{v}+v^{\prime}$, где $v^{\prime}>\bar{v}$. Отсюда

$$
\begin{aligned}
z= & u v^{-1}=\lambda X_{1}^{m_{1}} \ldots X_{n}^{m_{n}} \\
& \quad+\left[\left(\lambda X_{1}^{m_{1}} \ldots X_{n}^{m_{n}} \bar{v}+u^{\prime}\right)\left(\bar{v}+v^{\prime}\right)^{-1}-\lambda X_{1}^{m_{1}} \ldots X_{n}^{m_{n}}\right] \\
= & \lambda X_{1}^{m_{1}} \ldots X_{n}^{m_{n}}+\left(u^{\prime}-\lambda X_{1}^{m_{1}} \ldots X_{n}^{m_{n}} v^{\prime}\right) v^{-1} .
\end{aligned}
$$

Если $w=u^{\prime}-\lambda X_{1}^{m_{1}} \ldots X_{n}^{m_{n}} v^{\prime} \neq 0$, то по предложению 5.4

$$
\begin{aligned}
\pi\left(w v^{-1}\right) & =\pi(w)-\pi(v) \geqslant \min \left(\pi\left(u^{\prime}\right), \pi(z)+\pi\left(v^{\prime}\right)\right)-\pi(v) \\
& =\min \left(\pi\left(u^{\prime}\right)-\pi(v), \pi(z)+\pi\left(v^{\prime}\right)-\pi(v)\right) \\
& >\min (\pi(u)-\pi(v), \pi(z)+\pi(v)-\pi(v))=\pi(z) .
\end{aligned}
$$


Теорема 5.6. Пусть $C$-кольцо общих квантовьх многочленов из (7), $f \in F$, причем для некоторого ненулевого челого числа $d$ и некоторых $\lambda, \lambda^{\prime} \in D^{*}$, где $\lambda \lambda^{\prime} \in N$, выполнено равенство

$$
X_{1}^{d} f=\lambda f \lambda^{\prime} X_{1}^{d}
$$

Тогда $f$ лежит в подтеле $D\left(X_{1} ; \alpha_{1}\right)$, порожденном $D$ и $X_{1}$.

ДокАЗАТЕльство. Условие (22) эквивалентно тому, что

$$
\alpha_{1}^{d}(f)=\lambda f \lambda^{\prime} .
$$

Пусть $\pi(f)=\left(m_{1}, \ldots, m_{n}\right)$. Тогда в $D^{*} / N$ получаем

$$
\kappa\left(\alpha_{1}^{d}(f)\right)=\kappa(f) q_{12}^{d m_{2}} \ldots q_{1 n}^{d m_{n}} .
$$

Из (23) вытекает, что в $D^{*} / N$

$$
\kappa(f) q_{12}^{d m_{2}} \ldots q_{1 n}^{d m_{n}}=\kappa(f),
$$

и поэтому $m_{2}=\ldots=m_{n}=0$, так как $C$ - кольцо общих квантовых многочленов.

В силу предложения 5.4 имеем $f=X_{1}^{q} \lambda_{q}+z$, где $\lambda_{q} \in D^{*}, q \in \mathbb{Z}$ и $\pi(z)>\pi(f)$. Продолжая этот процесс, получаем представление $f$ в виде ряда:

$$
f=\sum_{i \geqslant q} X_{1}^{i} \lambda_{i} \in D\left[\left[X_{1} ; \alpha_{1}\right]\right], \quad \lambda_{i} \in D
$$

Без ограничения общности можно считать, что

$$
f=1+\sum_{i \geqslant 1} \lambda_{i} X_{1}^{i}=u^{-1} v
$$

где $u, v \in C$, причем

$$
u=1+\sum_{i=1}^{m} X_{1}^{i} b_{i}, \quad v=1+\sum_{j=1}^{m} X_{1}^{j} a_{j},
$$

где $b_{i}, a_{j}$ лежат в подкольце, порожденном $D, X_{2}, \ldots, X_{n}$. Таким образом, $v=u f$ и для любого $l \geqslant m+1$

$$
0=\lambda_{l}+\alpha_{1}^{1-l}\left(b_{1}\right) \lambda_{l-1}+\ldots+\alpha_{1}^{m-l}\left(b_{m}\right) \lambda_{l-m} .
$$

Пусть $b_{i}=\mu_{i}+b_{i}^{\prime}$, где $\mu_{i} \in D$ и $b_{i}^{\prime}$ - сумма одночленов вида

$$
\beta X_{1}^{l_{1}} \ldots X_{n}^{l_{n}}, \quad \beta \in D^{*}, \quad\left(l_{1}, \ldots, l_{n}\right) \in \mathbb{Z}^{n} \backslash\{(0, \ldots, 0)\} .
$$

Из (24) следует, что

$$
0=\lambda_{l}+\alpha_{1}^{1-l}\left(\mu_{1}\right) \lambda_{l-1}+\ldots+\alpha_{1}^{m-l}\left(\mu_{m}\right) \lambda_{l-m},
$$

и поэтому

$$
g=\left(1+\sum_{i=1}^{m} X_{1}^{i} \mu_{i}\right)^{-1} g \in D\left(X_{1} ; \alpha_{1}\right)
$$


Теорема 5.7. Пусть $C$-кольцо общих квантовых многочленов из $(7), f \in F$, причем для некоторого ненулевого челого числа $d$ и некоторых $\lambda, \lambda^{\prime} \in D^{*}$ выполнено равенство

$$
X_{1}^{d} f=\lambda f \lambda^{\prime} X_{1}^{d}
$$

Если $\pi(f)=\left(m_{1}, \ldots, m_{n}\right)$, то по модулю $N$

$$
\lambda \lambda^{\prime}=q_{12}^{d m_{1}} \ldots q_{1 n}^{d m_{n}}
$$

Kроме того, $f$ лежит в $D\left(X_{1} ; \alpha_{1}\right) X_{2}^{m_{2}} \ldots X_{n}^{m_{n}}$.

ДоКАЗАТЕЛЬСТво. Условие (25) эквивалентно условию (23). Как и в предыдушей теореме,

$$
\kappa\left(\alpha_{1}^{d}(f)\right)=\kappa(f) q_{12}^{d m_{1}} \ldots q_{1 n}^{d m_{n}}
$$

откуда вытекает (25). Кроме того, если $f^{\prime}=f X_{2}^{-m_{2}} \ldots X_{n}^{-m_{n}}$, то

$$
X_{1}^{d} f^{\prime}=\beta f^{\prime} \beta^{\prime} X_{1}^{d}, \quad \beta \beta^{\prime} \in N .
$$

Остается воспользоваться предыдушей теоремой.

Следствие 5.8. Пусть $C$ - кольцо общих квантовых многочленов из (7) и $\widehat{C}$ из предложения 5.3. Предположим, что задано вложение колеи $: \widehat{C} \rightarrow F$, причем $\phi(\widehat{D}) \subseteq D \quad$ u $\phi\left(Y_{1}\right)=\lambda_{1} X_{1}, \lambda_{1} \in D$. Тогда при $i \geqslant 2$

$$
\phi\left(Y_{i}\right)=a_{i} X_{2}^{m_{i 2}} \ldots X_{n}^{m_{i n}}, \quad a_{i} \in D\left(X_{1} ; \alpha_{1}\right), \quad m_{i j} \in \mathbb{Z}
$$

ДокАЗАТЕЛЬСТво. Пусть $i \geqslant 2$. Тогда $Y_{1} Y_{i}=\widehat{q_{1 i}} Y_{i} Y_{1}$, откуда

$$
\lambda_{1} X_{1} \phi\left(Y_{i}\right)=\phi\left(\widehat{q_{1 i}}\right) \phi\left(Y_{i}\right) \lambda_{1} X_{1}
$$

и поэтому

$$
X_{1} \alpha_{1}^{-1}\left(\lambda_{1}\right) \phi\left(Y_{2}\right)=\phi\left(\widehat{q_{12}}\right) \alpha^{-1}\left(\lambda_{1}^{-1}\right) \alpha_{1}^{-1}\left(\lambda_{1}\right) \phi\left(Y_{2}\right) \lambda_{1} X_{1}
$$

Остается воспользоваться предыдушей теоремой с

$$
d=1, \quad f=\alpha_{1}^{-1}\left(\lambda_{1}\right) \phi\left(Y_{i}\right), \quad \lambda=\phi\left(\widehat{q_{1 i}}\right) \alpha^{-1}\left(\lambda_{1}^{-1}\right), \quad \lambda^{\prime}=\lambda_{1} .
$$




\section{§ 6. Морита-эквивалентность}

Основная цель этого параграфа состоит в доказательстве теоремы о том, что если кольца обших квантовых многочленов Морита-эквивалентны, то они изоморфны. Кроме того, вычисляется группа Пикара кольца общих многочленов. Доказательство основано на изучении тел частных квантовых полиномов и колец эндоморфизмов проективных идеалов.

В начале параграфа рассматриваются свойства тел частных $F$ (см. (5)) кольца $A$ из (7). Пусть $K$ - подкольцо в $F$, причем сушествует такой ненулевой элемент $w \in \Lambda \backslash 0$, что $w K \subseteq \Lambda$. По условию $K \subseteq w^{-1} \Lambda$. Пусть $L$ - множество всех таких $a \in \Lambda$, что $w^{-1} a \in K$, т.е. $L=\Lambda \cap w K$. Так как $K$ является кольцом, то из условия $a, b \in L$ вытекает, что $a w^{-1} b \in L$, причем $w \in L$. Зафиксируем $b \in L \backslash 0$. По условию Оре найдутся такие элементы $u, v \in \Lambda \backslash 0$, что

$$
b u=w v .
$$

Таким образом, для любого $a \in L \backslash 0$ найдется такое $c \in L \backslash 0$, что

$$
a v=c u .
$$

Пусть $E$ - множество всех таких $\left(z_{1}, z_{2}\right) \in \Lambda \oplus \Lambda$, что $z_{1} v=z_{2} u$. По (27), (28) получаем $(a, c),(w, b) \in E$. Так как $b, w \neq 0$ и $\Lambda$ - область, то обе проекции $E$ в $\Lambda$ являются вложениями. Таким образом, $E$ изоморфно левому идеалу в $\Lambda$.

Пусть $(a, c) \in E$. В силу (27), (28) получаем $c^{-1} a=u v^{-1}=b^{-1} w$,т.е. $c=a w^{-1} b$.

Теорема 6.1. Eсли $n=1$, то $w=d^{\prime} w^{\prime}$ в $\Lambda$, причем $w^{\prime} K\left(w^{\prime}\right)^{-1} \subseteq \Lambda$.

ДоказАтЕльство. Выберем элемент $b \in E \backslash w \Lambda$. Так как $\Lambda$ - кольцо главных левых идеалов, то $E=\Lambda(p, q)$. Из определения $E$ вытекает, что наибольший левый обший делитель $p$ и $q$ равен 1 . Отсюда

$$
(w, b)=d(p, q), \quad(a, c)=f(p, q),
$$

где $d, f \in \Lambda$. При этом $d$ является наибольшим левым обшим делителем $w$ и $b$. Так как $b \notin \Lambda w$, то степень $d$ меньше степени $w$ и степень $p$ больше нуля. Так как $K$ - область, то из (27) вытекает, что $a=f p$ для некоторого $a \in L$. Следовательно, произвольный ненулевой элемент из $K$ имеет вид $w^{-1} a=p^{-1} d^{-1} f p$, где $d, p \in E \backslash 0$ фиксированы. Поэтому, переходя к подкольцу $p K p^{-1}$, можно уменшшить степень $w$. Таким образом, мы можем заменить $w$ на $d$. Совершая этот переход, мы окончательно получаем, что $w=d^{\prime} w^{\prime}$, где $d^{\prime}, w^{\prime} \in \Lambda$, причем $L=d^{\prime} \Lambda w^{\prime}$. Таким образом, если $a=d^{\prime} a^{\prime} w^{\prime} \in L$, то

$$
w^{-1} a=\left(w^{\prime}\right)^{-1}\left(d^{\prime}\right)^{-1} d^{\prime} a^{\prime} w^{\prime}=\left(w^{\prime}\right)^{-1} a^{\prime} w^{\prime} .
$$

Следовательно, $w^{\prime} K\left(w^{\prime}\right)^{-1}$ является подкольцом в $\Lambda$.

Рассмотрим теперь обший случай, когда $n \geqslant 1$.

Предложение 6.2. Если $z \in K \backslash 0 u \pi(z)=\left(m_{1}, \ldots, m_{n}\right) \in \mathbb{Z}^{n}, m_{o} m_{r+1}, \ldots, m_{n} \geqslant$ 0 
ДоКАЗАТЕЛЬСТвО. Пусть $i \geqslant r+1$ и $\Lambda_{i}-$ подкольцо в $\Lambda$, порожденное

$$
D, X_{1}^{ \pm 1}, \ldots, X_{r}^{ \pm 1}, X_{r+1}, \ldots, X_{i-1}, X_{i+1}, \ldots, X_{n} .
$$

Тогда $S_{i}=\Lambda_{i} \backslash 0$ удовлетворяет условию Оре. По теореме 6.1 сушествует такое

$$
w^{\prime} \in S_{i}^{-1} \Lambda=S_{i}^{-1} \Lambda_{i}\left[X_{i} ; \alpha_{i}\right], \quad w^{\prime} \neq 0,
$$

что $w^{\prime} K\left(w^{\prime}\right)^{-1} \subseteq S_{i}^{-1} \Lambda$. Если $z \in K \backslash 0$, то $\pi(z)=\pi\left(w^{\prime} z\left(w^{\prime}\right)^{-1}\right)$, откуда $m_{i} \geqslant 0$.

Всюду в дальнейшем мы будем предполагать, что $r \geqslant 1$. Как и в предложении 2.3 , совершая замену переменных вида

$$
X_{i} \rightarrow \begin{cases}X_{i} X_{1}^{-p^{i}}, & \text { если } i \geqslant 2, \\ X_{1}, & \text { если } i=1,\end{cases}
$$

для достаточно большого натурального числа $p$, можно считать, что в $w$ старший и младший коэффициенты по $X_{1}$ являются одночленами.

Пусть $\Lambda_{1}$ - подкольцо в $\Lambda$, порожденное

$$
D, X_{2}^{ \pm 1}, \ldots, X_{r}^{ \pm 1}, X_{r+1}, \ldots, X_{n} .
$$

Положим $S_{1}=\Lambda_{1} \backslash 0$. В этом случае

$$
S_{1}^{-1} \Lambda=S_{1}^{-1} \Lambda_{1}\left[X_{1}^{ \pm 1} ; \alpha_{1}\right] .
$$

Теорема 6.3. Пусть $K-$ подкольцо в теле $F$ из (5), причем $w K \subseteq \Lambda$ для некоторого әлемента $w \in \Lambda \backslash 0$, у которого стариий и младиий члень относительно $X_{1}$ являются одночленами. Пусть $K^{*}$ - группа обратимых әлементов в $K$. Тогда существует такой әлемент $w^{\prime} \in S_{1}^{-1} \Lambda \backslash 0$, что младиий и стариий коэффициенты $w^{\prime}$ относительно $X_{1}$ являются одночленами из $C$, причем если $x \in K^{*}$, mo

$$
w^{\prime} x\left(w^{\prime}\right)^{-1}=c X_{1}^{m_{1}} \ldots X_{r}^{m_{r}}, \quad c \in D^{*}, \quad m_{i} \in \mathbb{Z} .
$$

В частности,

$$
w^{\prime} x\left(w^{\prime}\right)^{-1} \in D \Longleftrightarrow \pi(x)=0 \Longleftrightarrow \rho(x)=0 .
$$

ДокАЗАТЕльСтво. По теореме 6.1 в $S_{1}^{-1} \Lambda$ имеется такое разложение $w=d^{\prime} w^{\prime}$, что

$$
w^{\prime} K\left(w^{\prime}\right)^{-1} \subseteq S_{1}^{-1} \Lambda .
$$

Без ограничения обшности можно считать, что младший коэффициент $d^{\prime}$ относительно $X_{1}$ равен 1, а младший член $w^{\prime}$ совпадает с младшим членом $w$ и является одночленом. Так как каждый элемент из $K^{*}$ обратим, то для любого $x \in K^{*}$ элемент $y=w^{\prime} x\left(w^{\prime}\right)^{-1}$ обратим в $S_{1}^{-1} \Lambda$. Таким образом,

$$
y=X_{1}^{m} \lambda, \quad \lambda \in S_{1}^{-1} \Lambda_{1}, \quad m \in \mathbb{Z} .
$$


Но $w x=w\left(w^{\prime}\right)^{-1} x w^{\prime}=d^{\prime} x w^{\prime} \in \Lambda$. Пусть

$$
\begin{array}{ll}
d^{\prime}=X_{1}^{q}+\sum_{i>q} X_{1}^{i} a_{i}, & a_{i} \in S_{1}^{-1} \Lambda_{1}, \\
w^{\prime}=X_{1}^{t} b+\sum_{i>t} X_{1}^{i} b_{i}, \quad b_{i} \in S_{1}^{-1} \Lambda_{1},
\end{array}
$$

где $b \in \Lambda$ - одночлен от $X_{2}, \ldots, X_{n}$. Тогда младший коэффициент $w x$ относительно $X_{1}$ лежит в $\Lambda$ и равен $\alpha_{1}^{t}(\lambda) b$. Таким образом, $\lambda=h b_{1}^{-1}$, где $b_{1}=\alpha_{1}^{-t}(b) \in \Lambda_{1}$ является одночленом от $X_{2}^{ \pm 1}, \ldots, X_{r}^{ \pm 1}, X_{r+1}, \ldots, X_{n}$ и $h \in \Lambda$. По предложению 6.2 получаем, что

$$
\pi(\lambda)=\pi(h)-\pi\left(b_{1}\right)=\left(0, m_{2}, \ldots, m_{n}\right),
$$

где $m_{r+1}, \ldots, m_{n} \geqslant 0$. Таким образом, $\lambda \in A$ является суммой одночленов

$$
\theta X_{2}^{d_{2}} \ldots X_{n}^{d_{n}}, \quad \theta \in D^{*}, \quad d_{i} \in \mathbb{Z}
$$

причем $d_{r+1}, \ldots, d_{n} \geqslant 0$. Следовательно, $\lambda \in \Lambda \cap S_{1}^{-1} \Lambda_{1}$. Проводя аналогичные рассуждения для $x^{-1}$, получаем, что $\lambda \in \Lambda \cap S_{1}^{-1} \Lambda_{1}$. Итак, $\lambda$ - обратимый элемент из $\Lambda \cap S_{1}^{-1} \Lambda_{1}$, т.е. $\lambda$ является одночленом от $X_{2}^{ \pm 1}, \ldots, X_{r}^{ \pm 1}$.

Следствие 6.4. Пусть $\widehat{D}-$ подтело в $K$. Тогда $\widehat{D} \subseteq\left(w^{\prime}\right)^{-1} D w^{\prime}$.

Теорема 6.5. Пусть $A$ - произвольное кольцо лорановских квантовых многочленов из (7), $\widehat{D}-$ тело с набором автоморфизмов

$$
\widehat{\alpha}=\left(\widehat{\alpha}_{1}, \ldots, \widehat{\alpha}_{m}\right),
$$

где $\widehat{Q}=\left(\widehat{q}_{i j}\right) \in \operatorname{Mat}(m, \widehat{D})$ u $\widehat{A}=\widehat{D}_{\widehat{Q}, \widehat{\alpha}}\left[Y_{1}^{ \pm 1}, \ldots, Y_{m}^{ \pm 1}\right]$.

Предположим, что задано вложение колеи $\phi: \widehat{A} \rightarrow F$, причем существует такой ненулевой әлемент $w \in A \cap \phi(\widehat{A})$, что $A w \phi(\widehat{A}) \subseteq A \cap \phi(\widehat{A}), u \phi(\widehat{A}) \phi(\widehat{A} \backslash$ $0)^{-1}=F$. Тогда $m=n$ и существует такой әлемент $w^{\prime} \in F$, что после некоторой замены переменных $Y_{1}, \ldots, Y_{n} \quad u \quad X_{1}, \ldots, X_{n}$

$$
w^{\prime} \phi(\widehat{D})\left(w^{\prime}\right)^{-1}=D, \quad w^{\prime} \phi\left(Y_{i}\right)\left(w^{\prime}\right)^{-1}=X_{i}, \quad 1 \leqslant i \leqslant n .
$$
A.

В частности, отображсние $\psi(z)=w^{\prime} \phi(z)\left(w^{\prime}\right)^{-1}$ задает изоморфизм $\psi: \widehat{A} \rightarrow$

ДокАЗАТЕЛЬСтво. Заметим, что $A$ и $\widehat{A}$ равноправны, ибо от $\phi$ можно перейти к $\phi^{-1}$. Поэтому можно считать, что $n \geqslant m$. В силу теоремы 6.3 сушествует такой элемент $w^{\prime}$, что $\psi(\widehat{D})=w^{\prime} \phi(\widehat{D})\left(w^{\prime}\right)^{-1} \subseteq D$ и каждое $\psi\left(Y_{i}\right)=w^{\prime} \phi\left(Y_{i}\right)\left(w^{\prime}\right)^{-1}, i \geqslant 1$, является одночленом от $X_{1}, \ldots, X_{n}$. В силу предложения 5.3 получаем $m=n$ и $\psi(\widehat{D})=D$. Кроме того, так как соответствуюшая матрица $\Delta$ из (18) лежит в $\mathrm{GL}(n, \mathbb{Z})$, то, совершая замену переменных $Y_{1}, \ldots, Y_{n}$ и $X_{1}, \ldots, X_{n}$, можно считать, что $\Delta$ - единичная матрица. Так как $\psi(\widehat{D})=D$, то, делая линейную замену $Y_{1}, \ldots, Y_{n}$, можно добиться, чтобы

$$
w^{\prime} \phi\left(Y_{i}\right)\left(w^{\prime}\right)^{-1}=X_{i}, \quad 1 \leqslant i \leqslant n .
$$


ЗАМЕчАНИЕ 6.6 . Подкольцо $\phi(\widehat{A})$ в теле $F$ является правым порядком и правым дробным идеалом в смысле [14, п. 3.1 .2 , с. 62 ; п. 3.1 .11$, с. 65$]$.

Теорема 6.7. Пусть $\Lambda$ - кольцо общих квантовых многочленов с $r \geqslant 1, \widehat{D}-$ тело с набором автоморфизмов $\hat{\alpha}=\left(\widehat{\alpha}_{1}, \ldots, \widehat{\alpha}_{m}\right), \widehat{Q}=\left(\widehat{q}_{i j}\right) \in \operatorname{Mat}(m, \widehat{D}) u$

$$
\widehat{\Lambda}=\widehat{D}_{\widehat{Q}, \widehat{\alpha}}\left[Y_{1}^{ \pm 1}, \ldots, Y_{s}^{ \pm 1}, Y_{s+1}, \ldots, Y_{m}\right]
$$

- другое кольцо общих квантовых многочленов, $s \geqslant 1$. Предположим, что задано вложение колеи $\phi: \widehat{\Lambda} \rightarrow F$, причем

$$
\phi(\widehat{A}) \phi(\widehat{A} \backslash 0)^{-1}=F
$$

и существует такой әлемент $w \in \Lambda \backslash 0$, что $w \phi(\widehat{\Lambda}) \subseteq \Lambda$. Предположим, что челочисленные векторь

$$
\pi\left(\phi\left(Y_{1}\right)\right), \ldots, \pi\left(\phi\left(Y_{s}\right)\right) \in \mathbb{Z}^{n}
$$

независимы. Тогда $s \leqslant r, n \leqslant m$ и существует такой әлемент $w^{\prime} \in F$, что

$$
w^{\prime} \phi(\widehat{D})\left(w^{\prime}\right)^{-1} \subseteq D, \quad w^{\prime} \phi(\widehat{\Lambda})\left(w^{\prime}\right)^{-1} \subseteq \Lambda,
$$

причем после некоторой замены переменных $Y_{1}, \ldots, Y_{s}$ u $X_{1}, \ldots, X_{r}$ получаем, что при $n \geqslant 2$ u $i=1, \ldots, s$

$$
w^{\prime} \phi\left(Y_{i}\right)\left(w^{\prime}\right)^{-1}=\lambda_{i} X_{i}, \quad \lambda_{i} \in D^{*}
$$

Если же либо $s \geqslant 2$, либо $s=1, m>2$, то $w^{\prime} \phi\left(Y_{i}\right)\left(w^{\prime}\right)^{-1}$ при $i \geqslant s+1$ является одночленом от $X_{s+1}^{ \pm 1}, \ldots, X_{r}^{ \pm 1}, X_{r+1}, \ldots, X_{n}$. Ecли жсе $s=1, m=2$, то при $n=$ 2

$$
w^{\prime} \phi\left(Y_{1}\right)\left(w^{\prime}\right)^{-1}=X_{1}, \quad w^{\prime} \phi\left(Y_{2}\right)\left(w^{\prime}\right)^{-1}=a X_{2}, \quad w^{\prime} \phi(\widehat{D})\left(w^{\prime}\right)^{-1}=D
$$

əде $a \in D\left[X_{1}^{ \pm 1} ; \alpha_{1}\right]$. Eсли жсе $s=r=n=1, m=2$, mo

$$
\begin{gathered}
w^{\prime} \phi\left(Y_{1}\right)\left(w^{\prime}\right)^{-1}=\lambda_{1} X_{1}^{d}, \quad \lambda \in D^{*}, \quad d \in \mathbb{Z}, \\
w^{\prime} \phi\left(Y_{2}\right)\left(w^{\prime}\right)^{-1} \in w^{\prime} \phi(\widehat{D})\left(w^{\prime}\right)^{-1} \cap D\left[X_{1}^{ \pm 1} ; \alpha_{1}\right], \\
w^{\prime} \phi(\widehat{D})\left(w^{\prime}\right)^{-1} \varsubsetneqq D .
\end{gathered}
$$


ДоКАЗАТЕЛЬСТво. По теореме 6.5 сушествует такой элемент $w^{\prime} \in S^{-1} \Lambda$, что

$$
w^{\prime} \phi\left(\widehat{\Lambda}^{*}\right)\left(w^{\prime}\right)^{-1} \subseteq \Lambda^{*}, \quad w^{\prime} \phi(\widehat{\Lambda})\left(w^{\prime}\right)^{-1} \subseteq S^{-1} \Lambda .
$$

В частности,

$$
w^{\prime} \phi(\widehat{D})\left(w^{\prime}\right)^{-1} \subseteq D
$$

Для любого $z \in \widehat{\Lambda}$ положим $\psi(z)=w^{\prime} \phi(z)\left(w^{\prime}\right)^{-1}$. Совершая замены переменных, можно считать, что при $i=1, \ldots, s$

$$
\psi\left(Y_{i}\right)=\lambda_{i} X_{i}^{d_{i}}, \quad \lambda_{i} \in D^{*}, \quad d_{i} \in \mathbb{Z} .
$$

По условию $d_{i} \neq 0$. Поэтому $s \leqslant r$. В силу следствия 5.8 и (31) для $i \geqslant s+1$ получаем

$$
\psi\left(Y_{i}\right)=a_{i} X_{2}^{m_{i 2}} \ldots X_{n}^{m_{i n}} \in S^{-1} \Lambda, \quad a_{i} \in D\left(X_{1} ; \alpha_{1}\right) .
$$

Тогда $a_{i} \in D\left[X^{ \pm 1} ; \alpha_{1}\right]$. Покажем, что если либо $s \geqslant 2$, либо $s=1, m>2$, то $a_{i}\left(X_{1}\right)$ является одночленом от $X_{1}$. Если $s \geqslant 2$, то, переставляя $X_{1}$ и $X_{2}$ местами, по тем же соображениям для любого $i \geqslant s+1$ получаем

$$
\psi\left(Y_{i}\right)=a_{i}^{\prime} X_{1}^{m_{i 1}^{\prime}} X_{3}^{m_{i 3}^{\prime}} \ldots X_{n}^{m_{i n}^{\prime}}, \quad a_{i}^{\prime} \in D\left[X_{2}^{ \pm 1} ; \alpha_{2}\right] .
$$

Отсюда для всех $i \geqslant s+1$

$$
\psi\left(Y_{i}\right)=\mu_{i} X_{1}^{l_{i 1}} X_{2}^{l_{i 2}} \ldots X_{n}^{l_{i n}}, \quad l_{i j} \in \mathbb{Z}, \quad \mu_{i} \in D^{*},
$$

и в этом случае утверждение доказано.

Пусть $s=1$. Воспользуемся предложением 5.3, где вместо $\widehat{D}$ возьмем $\widehat{D}\left(Y_{1} ; \widehat{\alpha}_{1}\right)$. Из (33) в силу предложения 5.3 получаем, что матрица

$$
\Delta^{\prime}=\left(m_{i j}\right) \in \operatorname{Mat}((m-1) \times(n-1), \mathbb{N} \cup 0)
$$

задает эпиморфизм $\Delta^{\prime}: \mathbb{Z}^{m-1} \rightarrow \mathbb{Z}^{n-1}$. Отсюда вытекает, что столбцы $\Delta^{\prime}$ линейно независимы над $\mathbb{Q}$. Кроме того, $m \geqslant n$.

Пусть $s=1, n \geqslant 3$. Тогда

$$
\psi\left(Y_{2}\right) \psi\left(Y_{3}\right)=\psi\left(\widehat{q}_{12}\right) \psi\left(Y_{3}\right) \psi\left(Y_{2}\right) .
$$

В силу (33)

$$
a_{2} X_{2}^{m_{22}} \ldots X_{n}^{m_{2 n}} a_{3} X_{2}^{m_{32}} \ldots X_{n}^{m_{3 n}}=\psi\left(\widehat{q}_{12}\right) a_{3} X_{2}^{m_{32}} \ldots X_{n}^{m_{3 n}} a_{2} X_{2}^{m_{22}} \ldots X_{n}^{m_{2 n}} .
$$

Пусть

$$
\pi\left(a_{i}\right)=\left(n_{i}, 0, \ldots, 0\right), \quad \rho\left(a_{i}\right)=\left(d_{i}, 0, \ldots, 0\right) .
$$

Тогда $d_{i} \geqslant n_{i}$. Положим

$$
x=\left(n_{2}, m_{22}, \ldots, m_{2 n}\right), y=\left(n_{3}, m_{32}, \ldots, m_{3 n}\right) \in \mathbb{Z}^{n} .
$$


Тогда в группе $G$

$$
\kappa\left(a_{2}\right) \kappa\left(a_{3}\right) \mu(x, y)=\psi\left(\widehat{q}_{12}\right) \kappa\left(a_{2}\right) \kappa\left(a_{3}\right) \mu(y, x),
$$

откуда

$$
\psi\left(\widehat{q}_{12}\right)=\mu(x, y) \mu(y, x)^{-1} .
$$

Аналогично, рассматривая старший член, получаем, что

$$
\mu(x, y) \mu(y, x)^{-1}=\mu\left(d, d^{\prime}\right) \mu\left(d^{\prime}, d\right)^{-1},
$$

где

$$
d=\left(d_{2}, m_{22}, \ldots, m_{2 n}\right), d^{\prime}=\left(d_{3}, m_{32}, \ldots, m_{3 n}\right) \in \mathbb{Z}^{n} .
$$

Так как $\Lambda$ - кольцо общих многочленов, то, сравнивая показатели при $q_{1 j}, 1<j \leqslant n$, получаем

$$
-n_{3} m_{2 j}+n_{2} m_{3 j}=-d_{3} m_{2 j}+d_{2} m_{3 j} .
$$

Отсюда при $j=2, \ldots, n$

$$
\left(d_{3}-n_{3}\right) m_{2 j}=\left(d_{2}-n_{2}\right) m_{3 j} \geqslant 0 .
$$

По доказанному выше все столбцы матрицы $\Delta^{\prime}=\left(m_{i j}\right)$, где $i, j \geqslant 2$, независимы. Поэтому из (34) получаем $d_{3}=n_{3}, d_{2}=n_{2}$, т.е. $\psi\left(Y_{2}\right), \ldots, \psi\left(Y_{m}\right)$ являются одночленами от $X_{1}, \ldots, X_{n}$. Итак, если либо $s \geqslant 2$, либо $s=1, m \geqslant 3$, то соответствующая матрица $\Delta$ из предложения 5.3 имеет вид

$$
\left(\begin{array}{c|c}
\operatorname{diag}\left(d_{1}, \ldots, d_{s}\right) & 0 \\
\hline \star & \Delta^{\prime \prime}
\end{array}\right)
$$

и эта матрица задает эпиморфизм $\mathbb{Z}^{m} \rightarrow \mathbb{Z}^{n}$. Следовательно,

$$
\operatorname{diag}\left(d_{1}, \ldots, d_{s}\right) \in \mathrm{GL}(s, \mathbb{Z}),
$$

т.е. можно считать, что $d_{1}=\ldots=d_{s}=1$.

Пусть, наконец, $s=1, m=2$. По доказанному выше $n \leqslant 2$. Пусть $n=2$. Тогда

$$
\begin{aligned}
\psi(\widehat{D}) & \subseteq D, \quad \psi\left(Y_{1}\right)=\lambda_{1} X_{1}^{d_{1}}, \quad \psi\left(Y_{2}\right)=a X_{2}^{d_{2}}, \\
d_{i} & \in \mathbb{Z}, \quad d_{2} \geqslant 2, \quad \lambda_{1} \in D^{*}, \quad a \in D\left[X_{1} ; \alpha_{1}\right] .
\end{aligned}
$$

Воспользуемся предложением 5.3 , где вместо $D$ и $\widehat{D}$ возьмем соответственно $D\left(X_{1} ; \alpha_{1}\right)$ и $\widehat{D}\left(Y_{1} ; \widehat{\alpha}_{1}\right)$. Тогда $\psi\left(\widehat{D}\left(Y_{1} ; \widehat{\alpha}_{1}\right)=D\left(X_{1} ; \alpha_{1}\right)\right.$ и $d_{2}=1$, поскольку соответствуюшая матрица $\Delta=\left(d_{2}\right) \in \mathrm{GL}(1, \mathbb{Z})$.

В силу условия (30) сушествуют такие $f, g \in \widehat{C} \backslash 0$, что $X_{1} \psi(f)=\psi(g)$. Без ограничения обшности в силу (35) можно считать, что

$$
f=\delta Y_{1}^{l_{1}} Y_{2}^{l_{2}}, \quad g=\gamma Y_{1}^{s_{1}} Y_{2}^{s_{2}}, \quad \delta, \gamma \in \widehat{D}^{*} .
$$

Тогда

$$
X_{1} \psi(\delta)\left(\lambda_{1} X_{1}^{d_{1}}\right)^{l_{1}}\left(a X_{2}\right)^{l_{2}}=\psi(\gamma)\left(\lambda_{1} X_{1}^{d_{1}}\right)^{s_{1}}\left(a X_{2}\right)^{s_{2}}
$$


Следовательно, $l_{2}=s_{2}$ и $1+d_{1} l_{1}=d_{1} s_{1}$, откуда $d_{1}=s_{1}-l_{1}= \pm 1$. Переходя от $X_{1}$ к $X_{1}^{-1}$, можно считать, что $d_{1}=1$.

Если $\lambda \in D$, то существуют такие $f, g \in \widehat{C} \backslash 0$, что

$$
\lambda \psi(f)=\psi(g) .
$$

В силу (35) можно считать, что $f, g$ из (36). Тогда

$$
\lambda \psi(\delta)\left(\lambda_{1} X_{1}\right)^{l_{1}}\left(a X_{2}\right)^{l_{2}}=\psi(\gamma)\left(\lambda_{1} X_{1}\right)^{s_{1}}\left(a X_{2}\right)^{s_{2}},
$$

откуда $l_{1}=s_{1}, l_{2}=s_{2}$, и поэтому $\lambda \psi\left(\gamma \delta^{-1}\right) \in \psi(\widehat{D})$. Таким образом, $\psi(\widehat{D})=D$, и можно считать, что

$$
\psi\left(Y_{1}\right)=X_{1}, \quad \psi\left(Y_{2}\right)=a X_{2}, \quad a \in D\left[X_{1}^{ \pm 1} ; \alpha_{1}\right] .
$$

Предположим, наконец, что $s=r=n=1, m=2$. Тогда

$$
\psi\left(Y_{1}\right)=\lambda_{1} X_{1}^{d_{1}}, \quad d_{1} \in \mathbb{Z} \backslash 0, \quad \psi\left(Y_{2}\right)=a \in D\left[X_{1}^{ \pm 1} ; \alpha_{1}\right] .
$$

При этом

$$
\psi\left(Y_{1}\right) \psi\left(Y_{2}\right)=\psi\left(\widehat{q}_{12}\right) \psi\left(Y_{2}\right) \psi\left(Y_{1}\right),
$$

откуда $\lambda_{1} X_{1}^{d_{1}} a=\psi\left(\widehat{q}_{12}\right) a \lambda_{1} X_{1}^{d_{1}}$. Если $\bar{a}=\delta X_{1}^{d_{2}}$, то

$$
\lambda \alpha_{1}^{d_{1}}(\delta)=\psi\left(\widehat{q}_{12}\right) \delta \alpha_{1}^{d_{2}}(\lambda),
$$

т.е. $\psi\left(\widehat{q}_{12}\right) \in N$, что невозможно, если $\psi(\widehat{D})=D$.

Предложение 6.8. Пусть $\Lambda, \widehat{\Lambda}-$ кольца общих квантовых многочленов из теоремь 6.7, причем $s, r \geqslant 1$. Предположим, что задано вложение колеи $\phi: \widehat{\Lambda} \rightarrow$ $F$, причем выполнено (30), и существует такой ненулевой әлемент $w \in \Lambda \cap \phi(\widehat{\Lambda})$, чmo

$$
\Lambda w \phi(\widehat{\Lambda}) \subseteq \Lambda \cap \phi(\widehat{\Lambda}) .
$$

Тогда әлементы $\pi\left(\phi\left(Y_{1}\right)\right), \ldots, \pi\left(\phi\left(Y_{s}\right)\right) \in \mathbb{Z}^{n}$ независимы и найдется такой әлемент $w^{\prime} \in F^{*}$, что $w^{\prime} \phi(\widehat{D})\left(w^{\prime}\right)^{-1}=D$.

ДоКАЗАТЕЛЬСтво. По теореме 6.3 и следствию 6.4 существует такой элемент $w^{\prime} \in$ $F^{*}$, что $w^{\prime} \phi(\widehat{D})\left(w^{\prime}\right)^{-1} \subseteq D$ и $w^{\prime} \phi\left(Y_{i}\right)\left(w^{\prime}\right)^{-1}$ при $i=1, \ldots, s$ является одночленом от $X_{1}, \ldots, X_{r}$. Но $\phi^{-1}: \Lambda \rightarrow F$ и $\phi^{-1}(\Lambda) \phi^{-1}(w) \subseteq \widehat{\Lambda}$. Поэтому, применяя теорему 6.3 и следствие 6.4 , получаем

$$
w^{\prime \prime} \phi^{-1}(D)\left(w^{\prime \prime}\right)^{-1} \subseteq \widehat{D}
$$

для некоторого $w^{\prime \prime} \in F^{*}$.

Пусть $l_{1}, \ldots, l_{s} \in \mathbb{Z}_{\text {и }}$

$$
0=l_{1} \pi\left(\phi\left(Y_{1}\right)\right)+\ldots+l_{s} \pi\left(\phi\left(Y_{s}\right)\right)=\pi\left(\phi\left(Y_{1}^{l_{1}}\right) \ldots \phi\left(Y_{s}^{l_{s}}\right)\right) .
$$


По теореме 6.3 найдется такой элемент $w^{\prime} \in F$, что

$$
w^{\prime} \phi\left(Y_{1}^{l_{1}} \ldots Y_{s}^{l_{s}}\right)\left(w^{\prime}\right)^{-1}=\lambda X_{1}^{d_{1}} \ldots X_{r}^{d_{r}}, \quad \lambda \in D^{*}, \quad d_{i} \in \mathbb{Z} .
$$

Применяя $\pi$, получаем в $\mathbb{Z}^{n}$, что $\left(d_{1}, \ldots, d_{r}, 0, \ldots, 0\right)=0$, т.е. $d_{1}=\ldots=d_{r}=0$. Отсюда

$$
\phi\left(Y_{1}^{l_{1}} \ldots Y_{s}^{l_{s}}\right)=\left(w^{\prime}\right)^{-1} \lambda w^{\prime}, \quad \lambda \in D^{*} .
$$

Следовательно, по (39)

$$
Y_{1}^{l_{1}} \ldots Y_{s}^{l_{s}}=u^{-1} \lambda^{\prime} u, \quad u \in F^{*}, \quad \lambda^{\prime} \in \widehat{D} .
$$

Пусть $\hat{\pi}$ определено в $\widehat{\Lambda}$ аналогично $\pi$. Тогда из (40) в $\mathbb{Z}^{m}$ получаем

$$
\left(l_{1}, \ldots, l_{s}, 0, \ldots, 0\right)=\hat{\pi}\left(\lambda^{\prime}\right)=0,
$$

т.e. $l_{1}=\ldots=l_{s}=0$.

Теорема 6.9. Пусть $\Lambda, \widehat{\Lambda}, r, s \geqslant 1, n \geqslant m, \phi$ из теоремы 6.7, причем $\Lambda$ и $\widehat{\Lambda}-$ кольца общих квантовых многочленов. Предположим, что существует такой элемент

$$
w \in \Lambda \cap \phi(\widehat{\Lambda}), \quad w \neq 0,
$$

что выполнено условие (38). Тогда $s=r, n=m$ и существует такой әлемент $w^{\prime} \in S^{-1} \Lambda$, что после некоторой замены переменных $Y_{1}, \ldots, Y_{s}$ и $X_{1}, \ldots, X_{r}$ вьполнень равенства

$$
w^{\prime} \phi(\widehat{D})\left(w^{\prime}\right)^{-1}=D, \quad w^{\prime} \phi\left(Y_{i}\right)\left(w^{\prime}\right)^{-1}=X_{i}, \quad 1 \leqslant i \leqslant n .
$$

В частности, отображение $z \mapsto w^{\prime} \phi(z)\left(w^{\prime}\right)^{-1}$ задает изоморфизм колеи $\Lambda$ и $\widehat{\Lambda}$.

ДоКАЗАТЕльСтво. По теореме 6.7 и предложению 6.8 получаем, что $m=n$. Следовательно, по теореме 6.7 существует такой элемент $w^{\prime} \in S^{-1} \Lambda$, что после некоторой замены переменных

$$
w^{\prime} \phi(\widehat{D})\left(w^{\prime}\right)^{-1} \subseteq D, \quad w^{\prime} \phi\left(Y_{i}\right)\left(w^{\prime}\right)^{-1}=\lambda_{i} X_{i}, \quad \lambda_{i} \in D^{*}, \quad 1 \leqslant i \leqslant r,
$$

и каждое $w^{\prime} \phi\left(Y_{i}\right)\left(w^{\prime}\right)^{-1}$ является одночленом от $X_{1}, \ldots, X_{n}$. Как отмечено в предложении 6.8 , кольца $\Lambda$ и $\widehat{\Lambda}$ равноправны, и поэтому $r=s$. Кроме того, попредложению 5.3 получаем первое равенство в (41).

Если $i \geqslant s+1$ и

$$
w^{\prime} \phi\left(Y_{i}\right)\left(w^{\prime}\right)^{-1}=\lambda_{i} X_{1}^{m_{i 1}} \ldots X_{n}^{m_{i n}}
$$

то, заменяя $Y_{i}$ на $Y_{i}^{\prime}=Y_{r}^{-m_{i r}} \ldots Y_{1}^{-m_{i 1}} Y_{i}$, получаем

$$
w^{\prime} \phi\left(Y_{i}^{\prime}\right)\left(w^{\prime}\right)^{-1}=\lambda_{i} X_{r+1}^{m_{i, r+1}} \ldots X_{n}^{m_{i n}}, \quad m_{i j} \geqslant 0 .
$$

Как и в предложении 5.3 , матрица $\Delta$ имеет вид

$$
\Delta=\left(\begin{array}{c|c}
E_{s} & 0 \\
\hline 0 & \Delta^{\prime}
\end{array}\right), \quad \Delta^{\prime}=\left(m_{i j}\right)_{i, j>r} \in \mathrm{GL}(n-r, \mathbb{Z}),
$$


где $E_{r}$ - единичная матрица размера $r$. Аналогично найдется такое $w^{\prime \prime} \in F^{*}$, что для $i=1, \ldots, n$

$$
\left(w^{\prime \prime}\right)^{-1} \phi^{-1}\left(X_{i}\right) w^{\prime \prime}=\theta_{i} Y_{1}^{s_{i 1}} \ldots Y_{n}^{s_{i n}}, \quad \theta_{i} \in \widehat{D}
$$

причем

$$
S=\left(s_{i j}\right)=\Delta^{-1} \in \mathrm{GL}(n, \mathbb{Z})
$$

Следовательно,

$$
S=\left(\begin{array}{c|c}
E_{r} & 0 \\
\hline 0 & S^{\prime}
\end{array}\right), \quad S^{\prime}=\left(s_{i j}\right)_{i, j>r}=\left(\Delta^{\prime}\right)^{-1} \in \mathrm{GL}(n-r, \mathbb{Z}),
$$

причем $s_{i j} \geqslant 0$. Таким образом, матрицы $S^{\prime}, \Delta^{\prime}$ неотрицательны. Поэтому матрица $\Delta^{\prime}$ является перестановочной. Перенумеровывая $Y_{r+1}, \ldots, Y_{n}$, можно считать, что

$$
w^{\prime} \phi\left(Y_{i}\right)\left(w^{\prime}\right)^{-1}=X_{i} \quad \text { для всех } i=1, \ldots, n .
$$

Перейдем теперь к рассмотрению колец эндоморфизмов левых идеалов в $\Lambda$. Нам потребуется

Предложение 6.10. Пусть $I$ - ненулевой левьй идеал в $\Lambda$ и $K=\operatorname{End}_{\Lambda} I$. Тогда имеется вложение $\phi: K \rightarrow F$, причем правое тело частных $K$ совпадает $c F . B$ частности, $K$ не имеет делителей нуля. Если $w \in I \cap C$, то $\Lambda w \phi(K) \subseteq \Lambda \cap \phi(K)$.

ДокаЗАТЕЛьство. Пусть $w \in I \cap C$. Тогда $\Lambda w \subseteq I$. Если $a \in I \backslash 0$, то найдутся такие $u, v \in C \backslash 0$, что $u a=v w$. Если $\gamma \in K \backslash 0$, то $u \gamma(a)=v \gamma(w)$. Отсюда

$$
\gamma(a)=u^{-1} v \gamma(w)=a w^{-1} \gamma(w)
$$

Таким образом, если $\phi(\gamma)=w^{-1} \gamma(w) \in F$, то $\gamma(a)=a \phi(\gamma)$ для любого $a \in I$. Нетрудно видеть, что сопоставление $\phi$ является вложением $K$ в $F$. При этом $w \phi(K) \subseteq \Lambda, w \in$ $\phi(K)$. Если $u, v \in C \backslash 0$, то

$$
v u^{-1}=(v w)(u w)^{-1} \in K \cdot(K \backslash 0)^{-1} .
$$

Теорема 6.11. Пусть $A, \widehat{A}-$ кольиа лорановских многочленов из теоремы 6.5. Предполохим, что существует такой левьй идеал $I$ в $A$, что $\operatorname{End}_{A} I \simeq \widehat{A}$. Тогда $A \simeq \widehat{A}$.

ДоКАЗАТЕЛЬСТво. В силу теоремы 6.3 и предложения 6.10 получаем, что выполнены все условия теоремы 6.5. Остается воспользоваться утверждением теоремы 6.5.

Теорема 6.12. Пусть I - проективный левый идеал в кольце общих квантовых многочленов $\Lambda$, где $r \geqslant 1$. Если $\operatorname{End}_{\Lambda} I \simeq \widehat{\Lambda}$, где $\widehat{\Lambda}-$ кольцо общих квантовых многочленов $c s \geqslant 1$ из теоремы 6.7, то I- главный левый идеал в $\Lambda$. Кроме moro, $\Lambda \simeq \widehat{\Lambda}$. 
ДокАзАТЕЛЬство. Пусть $w \in I \backslash 0$. Как показано в предложении 6.10 , сушествует такое вложение $\phi: \widehat{\Lambda} \rightarrow F$, что выполнены условия (38), (30). Так как $\Lambda, \widehat{\Lambda}$ равноправны, то можно считать, что $n \geqslant m$. По теореме 6.9 найдется такой элемент $w^{\prime} \in F^{*}$, что отображение $\psi$, где $\psi(z)=w^{\prime} \phi(z)\left(w^{\prime}\right)^{-1}$, является изоморфизмом колец, причем выполнены условия (41). Если $x \in I, z \in \Lambda$ и $z=\psi(y)$, то

$$
x\left(w^{\prime}\right)^{-1} z=x\left(w^{\prime}\right)^{-1} w^{\prime} \phi(y)\left(w^{\prime}\right)^{-1}=x \phi(y)\left(w^{\prime}\right)^{-1} \in I\left(w^{\prime}\right)^{-1} .
$$

Таким образом, $I\left(w^{\prime}\right)^{-1}$ является $(\Lambda-\Lambda)$-бимодулем и $I\left(w^{\prime}\right)^{-1} \cap \Lambda$ является ненулевым идеалом в $\Lambda$. Так как кольцо $A$ просто, то $I\left(w^{\prime}\right)^{-1} \supseteq \Lambda X_{r+1}^{d} \ldots X_{n}^{d}$ для некоторого $d \geqslant 0$. Следовательно, $X_{r+1}^{d} \ldots X_{n}^{d} w^{\prime} \in I$. Пусть $J$ - множество всех таких $u \in \Lambda$, что $I\left(w^{\prime}\right)^{-1} u \subseteq \Lambda$.

Лемма 6.13. $Ј$ является ненулевым идеалом в $\Lambda$.

ДокАЗАтЕльство. Пусть $r \in I$ и $a, b \in \Lambda$. Тогда $I\left(w^{\prime}\right)^{-1} a u b \subseteq I\left(w^{\prime}\right)^{-1} u b \subseteq \Lambda b \subseteq$ $\Lambda$. Так как кольцо $A=\Lambda_{X_{r+1} \ldots X_{n}}$ просто, то $J$ содержит $X_{r+1}^{l} \ldots X_{n}^{l}$ для некоторого $l \geqslant 0$. Положим

$$
L=I\left(w^{\prime}\right)^{-1} X_{r+1}^{l} \ldots X_{n}^{l} \subseteq \Lambda .
$$

Тогда $L$ является левым идеалом, изоморфным $I$. Кроме того, для любого $a \in \Lambda$

$$
\begin{aligned}
L a & =I\left(w^{\prime}\right)^{-1} X_{r+1}^{l} \ldots X_{n}^{l} a\left(X_{r+1}^{l} \ldots X_{n}^{l}\right)^{-1}\left(X_{r+1}^{l} \ldots X_{n}^{l}\right) \\
& \subseteq I\left(w^{\prime}\right)^{-1} X_{r+1}^{l} \cdots X_{n}^{l}=L
\end{aligned}
$$

поскольку $I\left(w^{\prime}\right)^{-1}$ является правым $\Lambda$-модулем и

$$
X_{r+1}^{l} \ldots X_{n}^{l} a\left(X_{r+1}^{l} \ldots X_{n}^{l}\right)^{-1} \in \Lambda
$$

Итак, можно считать, что $I=L$ - идеал в $\Lambda$. При этом $X_{r+1}^{q} \ldots X_{n}^{q} \in I$ для некоторого $q \geqslant 0$, ибо кольцо $A=\Lambda_{X_{r+1} \ldots X_{n}}$ просто

Для дальнейшего нам потребуется

Лемма 6.14. Пусть $\Lambda$ - кольцо общих квантовьх многочленов, $n>r$ и $I$-ненулевой проективный левый идеал в $\Lambda$, причем End $_{\Lambda} I=\Lambda$. Тогда $\operatorname{End}_{\Lambda / X_{n} \Lambda}\left(I / X_{n} I\right)=$ $\Lambda / X_{n} \Lambda$, m.e. $I X_{n} \subseteq X_{n} I$.

ДокАЗАТЕЛЬСТво. Заметим, что $I / X_{n} I$ является проективным модулем над кольцом $\Lambda / X_{n} \Lambda$. Кроме того, в силу проективности $I$ естественный эпиморфизм модулей $I \rightarrow I / X_{n} I$ индуцирует эпиморфизм колец эндоморфизмов

$$
\nu: \Lambda=\operatorname{End}_{\Lambda} I \rightarrow \operatorname{End}_{\Lambda / X_{n} \Lambda}\left(I / X_{n} I\right)
$$

Так как $I / X_{n} I$ изоморфно левому идеалу в $\Lambda / X_{n} \Lambda$ [1], то End $\Lambda_{/ X_{n} \Lambda}\left(I / X_{n} I\right)$ является областью. Следовательно, ker $\nu$ является первичным идеалом в $\Lambda$, и поэтому он содержит некоторое $X_{j}, j>r$. Следовательно, $\nu$ индуцирует эпиморфизм $D$-алгебр

$$
\Lambda / X_{j} \Lambda \simeq \Lambda / \operatorname{ker} \nu \rightarrow \Lambda / X_{n} \Lambda
$$


Так как $\Lambda$ - кольцо обших многочленов, то $q_{1 j}$ не лежит в подгруппе $D^{*}$, порожденной $N$ и всеми $q_{r s}$, где $1 \leqslant r<s \leqslant n$ и $r, s \neq j$. В силу [10, теорема 1.1] получаем $j=n$.

Завершим доказательство теоремы индукцией по $n-r$. Если $n=r$, то $I=\Lambda=A$, так как кольцо $A$ просто и $I$ - ненулевой идеал в $\Lambda$.

Пусть для числа не лорановских переменных, меньших $n-r$, утверждение верно. Взяв вместо $X_{n}$ в лемме 6.14 переменные $X_{r+1}, \ldots, X_{n}$, получаем, что для $i>r$

$$
I X_{i} \subseteq X_{i} I
$$

Заметим, что включение (42) выполнено и для $i=1, \ldots, r$, поскольку $I$ - двусторонний идеал в $\Lambda$. В частности, $I$ инвариантно относительно $\alpha_{1}^{-1}, \ldots, \alpha_{n}^{-1}$.

Лемма 6.15. Идеал I порождается одночленами от $X_{r+1}, \ldots, X_{n}$.

ДоКАЗАТЕЛЬСтво. Пусть

$$
f=\sum a_{i_{1}, \ldots, i_{n}} X_{1}^{i_{1}} \ldots X_{n}^{i_{n}} \in I \backslash 0, a_{i_{1}, \ldots, i_{n}} \in D
$$

и $X_{1}^{l_{1}} \ldots X_{n}^{l_{n}}$ - старший одночлен относительно лексикографического порядка. Применяя $\alpha_{j}^{-1}, 1 \leqslant j \leqslant n$, получаем

$$
\alpha_{j}^{-1}(f)=\sum b_{i_{1}, \ldots, i_{n}} X_{1}^{i_{1}} \ldots X_{n}^{i_{n}} \in I \backslash 0,
$$

где

$$
b_{i_{1}, \ldots, i_{n}}=\alpha_{j}^{-1}\left(a_{i_{1}, \ldots, i_{n}}\right) \lambda \cdot\left(\prod_{1 \leqslant s \leqslant n} q_{j s}^{-i_{s}}\right) \in D, \quad \lambda \in N .
$$

Тогда $I$ содержит элемент

$$
\begin{aligned}
& b_{l_{1}, \ldots, l_{n}} a_{l_{1}, \ldots, l_{n}}^{-1} f-\alpha_{j}^{-1}(f) \\
& \quad=\sum_{\left(i_{1}, \ldots, i_{n}\right)<\left(l_{1}, \ldots, l_{n}\right)}\left[b_{l_{1}, \ldots, l_{n}} a_{l_{1}, \ldots, l_{n}}^{-1} a_{i_{1}, \ldots, i_{n}}-b_{i_{1}, \ldots, i_{n}}\right] X_{1}^{i_{1}} \ldots X_{n}^{i_{n}} .
\end{aligned}
$$

Пусть $a_{i_{1}, \ldots, i_{n}} \neq 0$, но

$$
b_{l_{1}, \ldots, l_{n}} a_{l_{1}, \ldots, l_{n}}^{-1} a_{i_{1}, \ldots, i_{n}}=b_{i_{1}, \ldots, i_{n}} .
$$

Тогда в $D^{*} / N$ получаем

$$
\prod_{1 \leqslant s \leqslant n} q_{j s}^{-l_{s}}=\prod_{1 \leqslant s \leqslant n} q_{j s}^{-i_{s}}
$$

Отсюда

$$
\left(l_{1}, \ldots, l_{j-1}, l_{j+1}, \ldots, l_{n}\right)=\left(i_{1}, \ldots, i_{j-1}, i_{j+1}, \ldots, i_{n}\right) .
$$

Взяв $j=1$ и $j=n \geqslant 2$, получаем $\left(l_{1}, \ldots, l_{n}\right)=\left(i_{1}, \ldots, i_{n}\right)$, что невозможно. Итак, если $f$ не является одночленом, то в $I$ содержится элемент, являюшийся суммой меньшего числа одночленов, входящих в $f$. Индукционные соображения по числу одночленов показывают, что все одночлены, входящие в $f$, лежат в $I$. 
Без ограничения общности можно считать, что $I$ не лежит ни в каком $X_{j} \Lambda, j>r$. В частности, $I$ содержит элемент $X_{r+1}^{l_{r+1}} \ldots X_{n-1}^{l_{n-1}}$. По индукции модуль $I / X_{n} I$ порождается одним элементом. Следовательно, найдется такой элемент $e \in I$, что $I=\Lambda e+X_{n} I$. В частности,

$$
X_{r+1}^{l_{r+1}} \ldots X_{n-1}^{l_{n-1}}=a e+X_{n} g, \quad a \in \Lambda, \quad g \in I .
$$

Можно считать, что $а$ лежит в подкольце, порожденном

$$
D, X_{1}^{ \pm 1}, \ldots, X_{r}^{ \pm 1}, X_{r+1}, \ldots, X_{n-1}
$$

В этом случае (43) влечет $X_{r+1}^{l_{r+1}} \ldots X_{n-1}^{l_{n-1}}=a e$, т.е. $е$ по модулю $X_{n}$ является одночленом от $X_{r+1}, \ldots, X_{n-1}$. По лемме 6.15 элемент е является одночленом от $X_{r+1}, \ldots, X_{n-1}$.

Пусть $u=X_{n} v \in I$, где $v \in \Lambda$ - одночлен. Если $v \notin I$, то $X_{n} v=b e+X_{n} h$, $b \in \Lambda, h \in I$. Так как $e$ - одночлен, то по доказанному выше $b=X_{n} c, c \in \Lambda$. Отсюда $v=c e+h \in I$, и поэтому $X_{n} \Lambda \cap I=X_{n} I$. Но тогда $I=\Lambda e$.

Теорема 6.16. Пусть $\Lambda, \widehat{\Lambda}-$ кольца общих квантовых многочленов из теоремы 6.7, где $r, s \geqslant 1, n \geqslant 2$. Если $\Lambda$ и $\widehat{\Lambda}$ Морита-әквивалентны, то $\Lambda \simeq \widehat{\Lambda}$. Кроме того, $\operatorname{Pic}(\Lambda)$ тривиально.

ДокАЗАТЕЛЬСТво. По условию существует такой проективный $\Lambda$-модуль $I$, что $\operatorname{End}_{\Lambda} I \simeq \widehat{\Lambda}$. Если ранг $q$ модуля $I$ больше 1 , то $I \simeq \Lambda^{q}, q \geqslant 2$ [1]. В этом случае End $I \simeq \operatorname{Mat}(q, \Lambda)$, что невозможно, ибо $\operatorname{End}_{\Lambda} I \simeq \widehat{\Lambda}$. Итак, ранг $I$ равен 1 , т.е. $I$ изоморфно левому идеалу в $\Lambda[1],[9]$. Остается воспользоваться предыдушей теоремой.

Перейдем теперь к рассмотрению Морита-эквивалентности кольца общих квантовых многочленов $C$ из $(7)$ и кольца $\widehat{\Lambda}$ из теоремы 6.7 .

Теорема 6.17. Если $C$ и $\widehat{\Lambda}$ Морита-эквивалентны, то $s=0, n=m$.

ДокАЗАТЕЛЬСтво. Так как $C$ и $\widehat{\Lambda}$ - кольца общих квантовых многочленов, то кольца $A=C_{X_{1} \ldots X_{n}}$ и $\widehat{A}=\widehat{\Lambda}_{X_{1} \ldots X_{n}}$ просты [1]. Более того, как и в теореме 6.16 , сушествует такой проективный левый идеал $I$ в $C$, что $\operatorname{End}_{C} I \simeq \widehat{\Lambda}$. По [1] имеется изоморфизм $C$-модулей $I \oplus I \simeq C \oplus C$. Следовательно,

$$
\operatorname{Mat}(2, \widehat{\Lambda}) \simeq \operatorname{End}(I \oplus I) \simeq \operatorname{End}(C \oplus C) \simeq \operatorname{Mat}(2, C)
$$

Каждый идеал кольца матриц индуцирован некоторым идеалом кольца коэффициентов . В силу простоты $A$ и $\widehat{A}$ единственными минимальными первичными идеалами в кольце матриц $\operatorname{Mat}(2, \widehat{\Lambda})$ являются идеалы, порождаемые любой из матриц $Y_{s+1} E, \ldots, Y_{m} E$, где $E$ - единичная матрица. Аналогично, единственными минимальными первичными идеалами в $\operatorname{Mat}(2, C)$ являются идеалы, порождаемые любой из матриц $X_{1} E, \ldots, X_{n} E$. Поэтому $m-s=n$. Следовательно, если $s=0$, то теорема доказана.

Пусть $s \geqslant 1$. Тогда единственным максимальным первичным идеаломв $\operatorname{Mat}(2, \widehat{\Lambda})$ является идеал, порождаемый всеми матрицами $Y_{s+1} E, \ldots, Y_{m} E$. С другой стороны, этот идеал порождается всеми матрицами $X_{1} E, \ldots, X_{n} E$. Факторизуя по этому 
идеалу, можно считать, что $m=s, n=0$. Как известно, единственными нетривиальными левыми идеалами в $\operatorname{Mat}(2, D)$ являются левые идеалы вида

$$
\left(\begin{array}{l}
a \\
b
\end{array}\right)\left(\begin{array}{ll}
c & d
\end{array}\right)
$$

где $a, b \in D$ - произвольные элементы, $c, d \in D$ - фиксированная ненулевая пара. Таким образом, любая убываюшая цепь левых идеалов в $\operatorname{Mat}(2, D)$ имеет длину не более трех.

С другой стороны, в

$$
\operatorname{Mat}\left(s, \widehat{D}_{Q^{\prime} \alpha^{\prime}}\left[Y_{1}^{ \pm 1}, \ldots, Y_{s}^{ \pm 1}\right]\right), \quad s \geqslant 1,
$$

имеется счетная убывающая цепь левых идеалов

$$
\begin{aligned}
\ldots & \supset \operatorname{Mat}\left(2, \widehat{D}_{Q^{\prime} \alpha^{\prime}}\left[Y_{1}^{ \pm 1}, \ldots, Y_{s}^{ \pm 1}\right]\right)\left(1+Y_{1}\right)^{d} E \\
& \supset \operatorname{Mat}\left(2, \widehat{D}_{Q^{\prime} \alpha^{\prime}}\left[Y_{1}^{ \pm 1}, \ldots, Y_{s}^{ \pm 1}\right]\right)\left(1+Y_{1}\right)^{d+1} E \supset \ldots,
\end{aligned}
$$

где $d$ пробегает все натуральные числа. Отсюда $s=0$.

Теорема 6.18. Пусть $C$ - кольио общих квантовых многочленов из (7), Iпроективный левый идеал в $C$. Предположим, что $\operatorname{End}_{C} I$ изоморфен кольиу общих квантовых многочленов $\widehat{C}$ из предложсения 5.3, причем $n=m$. Тогда $I-$ главный левый идеал и $\widehat{C} \simeq C$.

ДОКАЗАТЕЛЬСтво. Случай $n=1$ очевиден. Поэтому можно считать, что $n>1$ и для $n-1$ теорема верна. По [1] имеем $I \oplus I=C e_{1} \oplus C e_{2}$, где

$$
e_{1}=(p, q), e_{2}=(r, s) \in I \oplus I .
$$

Если $\gamma \in \operatorname{End}_{C} I$, то $\gamma(x)=x \phi(\gamma) \in I$, где $\phi(\gamma) \in F$. Пусть $(x, y) \in C \oplus C$. Тогда

$$
\left(\begin{array}{ll}
x & y
\end{array}\right)\left(\begin{array}{l}
e_{1} \\
e_{2}
\end{array}\right) \gamma=\left(\begin{array}{ll}
x & y
\end{array}\right) A_{\gamma}\left(\begin{array}{l}
e_{1} \\
e_{2}
\end{array}\right)
$$

где $A_{\gamma} \in \operatorname{Mat}(2, C)$. Если $(u, v) \in I \oplus I$, то

$$
\left(\begin{array}{ll}
u & v
\end{array}\right) \gamma=\left(\begin{array}{ll}
u & v
\end{array}\right) A_{\gamma}^{\prime}, \quad A_{\gamma}^{\prime} \in \operatorname{Mat}(2, \phi(\widehat{C})) .
$$

Поэтому

$$
\left(\begin{array}{ll}
x & y
\end{array}\right)\left(\begin{array}{l}
e_{1} \\
e_{2}
\end{array}\right) \gamma=\left(\begin{array}{ll}
x & y
\end{array}\right)\left(\begin{array}{ll}
p & q \\
r & s
\end{array}\right) A_{\gamma}^{\prime}
$$

Отсюда

$$
A_{\gamma}^{\prime}=\left(\begin{array}{ll}
p & q \\
r & s
\end{array}\right)^{-1} A_{\gamma}\left(\begin{array}{ll}
p & q \\
r & s
\end{array}\right) .
$$


Как и в теореме 6.12 , можно считать, что $I / X_{n} I$ является свободным $\widehat{C} / Y_{n} \widehat{C}$-модулем. Таким образом, сушествует такой элемент $e_{1}^{\prime} \in I$, что $I=C e_{1}^{\prime}+X_{n} I$. Так как естественный гомоморфизм $\mathrm{GL}(2, C) \rightarrow \mathrm{GL}\left(2, C / X_{n} X\right)$ сюръективен, то можно считать, что

$$
\left(e_{1}^{\prime}, 0\right) \equiv e_{1} \quad \bmod X_{n}(I \oplus I), \quad\left(0, e_{1}^{\prime}\right) \equiv e_{2} \quad \bmod X_{n}(I \oplus I)
$$

Но тогда

$$
\left(\begin{array}{ll}
p & q \\
r & s
\end{array}\right) \equiv E \bmod X_{n}
$$

Кроме того, при $i=1, \ldots, n-1$

$$
A_{Y_{i}}^{\prime} \equiv\left(\begin{array}{cc}
X_{i} & 0 \\
0 & X_{i}
\end{array}\right) \quad \bmod X_{n}
$$

и для $i=1, \ldots, n$

$$
X_{i}(0, v) A_{Y_{i}}^{\prime}=X_{i}\left(0, v_{i}^{\prime}\right), \quad X_{i}(u, 0) A_{Y_{i}}^{\prime}=X_{i}\left(u_{i}^{\prime}, 0\right)
$$

Более того, если $\lambda \in D$, то

$$
A_{\lambda}^{\prime} \equiv\left(\begin{array}{cc}
\phi(\lambda) & 0 \\
0 & \phi(\lambda)
\end{array}\right) \quad \bmod X_{n}
$$

Итак,

$$
A_{\lambda}^{\prime}=\left(E+X_{n} \Gamma^{\prime}\right)^{-1} A_{\gamma}\left(E+X_{n} \Gamma^{\prime}\right) .
$$

Отсюда

$$
\begin{aligned}
A_{Y_{i}}^{\prime} & =\left(E+X_{n} \Gamma^{\prime \prime}\right)\left(\operatorname{diag}\left(X_{i}, X_{i}\right)+\Delta^{\prime}\right)\left(E+X_{n} \Gamma^{\prime}\right) \\
& =X_{i}(u, 0)\left[\left(\begin{array}{cc}
X_{i} & 0 \\
0 & X_{i}
\end{array}\right)+X_{n} \Delta_{i}^{\prime}\right]=\left(X_{i} u X_{i}, 0\right)+X_{i}(u, 0) X_{n} \Delta^{\prime} \\
& =\left(X_{i} u X_{i}, 0\right)+\left(X_{i} u, 0\right)\left(\begin{array}{cc}
X_{n} z_{1} & X_{n} z_{2} \\
X_{n} z_{3} & X_{n} z_{4}
\end{array}\right) \\
& =\left(X_{i} u X_{i}, 0\right)+\left(X_{i} u X_{n} z_{1}, X_{i} u x_{n} z_{2}\right) .
\end{aligned}
$$

Следовательно, при $1 \leqslant i \leqslant n-1$

$$
A_{Y_{i}}^{\prime}=\left(\begin{array}{cc}
X_{i}+X_{n} z_{i 1} & 0 \\
0 & X_{i}+X_{n} z_{i 2}
\end{array}\right) ; \quad A_{Y_{n}}^{\prime}=\left(\begin{array}{cc}
X_{n} z_{n 1} & 0 \\
0 & X_{n} z_{n 2}
\end{array}\right) .
$$

Таким образом, учитывая (45), получаем

$$
\left(\begin{array}{cc}
p & q \\
r & s
\end{array}\right)\left(\begin{array}{cc}
X_{i}+X_{n} z_{i 1} & 0 \\
0 & X_{i}+X_{n} z_{i 2}
\end{array}\right)=\left(\begin{array}{cc}
X_{i} & 0 \\
0 & X_{i}
\end{array}\right)\left(\begin{array}{cc}
p & q \\
r & s
\end{array}\right)
$$


т.e.

$$
\left(\begin{array}{cc}
p\left(X_{i}+X_{n} z_{i 1}\right) & q\left(X_{i}+X_{n} z_{i 2}\right) \\
r\left(X_{i}+X_{n} z_{i 1}\right) & s\left(X_{i}+X_{n} z_{i 2}\right)
\end{array}\right)=\left(\begin{array}{cc}
X_{i} p & X_{i} q \\
X_{i} r & X_{i} s
\end{array}\right) .
$$

Но $r=X_{n}^{d} r^{\prime}, d \geqslant 1$. Поэтому $X_{n}^{d} r^{\prime}\left(X_{i}+X_{n} z_{i 1}\right)=X_{i} X_{n}^{d} r^{\prime}$. Отсюда $r^{\prime}\left(X_{i}+X_{n} z_{i 1}\right)=$ $q_{n i}^{-d} X_{i} r^{\prime}$. Таким образом, в $C / X_{n} C$ получаем $r^{\prime} X_{i}=q_{n i}^{-d} X_{i} r^{\prime}$. По теореме 5.7 это возможно лишь при $r^{\prime} \in X_{n} C$, т.е. $r=X_{n}^{d+1} r^{\prime}$. Следовательно, $r \in \bigcap_{d \geqslant 1} X_{n}^{d} C=0$. Аналогично получаем, что $s=0$, т.е.

$$
\Gamma=\left(\begin{array}{cc}
p & 0 \\
0 & s
\end{array}\right) \equiv E \bmod X_{n}
$$

В этом случае $e_{1}, e_{2} \in I$ и $C e_{1}, C e_{2} \subseteq I$. Но $I \oplus I=C e_{1} \oplus C e_{2}$. Поэтому $I=C e_{1}$ и $\widehat{C}=C$.

\section{§ 7. Проблема Зарисского}

Пусть $k$-поле и $k\left[X_{1}, \ldots, X_{n}\right]$ - кольцо многочленов. Предположим, что существует такое подкольцо $K$ в $k\left[X_{1}, \ldots, X_{n}\right]$, что $k\left[X_{1}, \ldots, X_{n}\right]$ изоморфно кольцу многочленов от одной переменной с коэффициентами в $K$. Гипотеза Зарисского утверждает, что $K$ изоморфно $k\left[X_{1}, \ldots, X_{n-1}\right]$. В обшем случае эта проблема не решена. В этом параграфе излагается решение аналога этой проблемы для общих квантовых многочленов. Всюду в этом параграфе $\Lambda$ обозначает кольцо общих квантовых многочленов.

Теорема 7.1 (проблема Зарисского для многочленов). Пусть $B-$ подкольцо в $\Lambda$, содержащее $D, Y \in \Lambda$, причем существует такой автоморфизм $\beta$ в $B$, что $Y b=\beta(b) Y$ для всех $b \in B$. Если $\Lambda=B[Y, \beta]$, то $Y=\lambda_{i} X_{i}$ для некоторого $i>r$, где $\lambda_{i} \in \Lambda^{*}$ - одночлен от $X_{1}, \ldots, X_{r}$ и $B$ совпадает с подкольцом, порожсденньм $D, X_{1}^{ \pm 1}, \ldots, X_{r}^{ \pm 1}$, а также со всеми $X_{j}, j>r, j \neq i$.

ДокАЗАТЕЛьство. Заметим, что $Y \Lambda$ является минимальным ненулевым вполне простым идеалом в $\Lambda$. Следовательно, $Y \Lambda$ совпадает с идеалом, порождаемым одной из переменных $X_{i}, i>r$. Пусть для простоты $Y \Lambda=X_{n} \Lambda$. В этом случае $Y=\lambda X_{n}$, где $\lambda-$ обратимый элемент из $\Lambda$, т.е. $\lambda$ - одночлен от $X_{1}, \ldots, X_{r}$. Таким образом,

$$
B \simeq \Lambda / Y \Lambda \simeq \Lambda^{\prime}=D_{*, *}\left[X_{1}^{ \pm 1}, \ldots, X_{r}^{ \pm 1}, X_{r+1}, \ldots, X_{n-1}\right]
$$

Пусть $\phi: \Lambda^{\prime} \rightarrow B \subseteq \Lambda$ - соответствуюшее вложение. Тогда

$$
\phi(z)=z+X_{n} \psi(z), \quad \psi(z) \in \Lambda \text {. }
$$

В частности, $\phi(D)$ является подтелом в $\Lambda$. Но $D$ содержит все подтела в $\Lambda$. Поэтому $\phi(D)=D$.

Пусть $j=1, \ldots, n-1$. Тогда

$$
\begin{aligned}
Y X_{j} Y^{-1} & =\lambda X_{n} Y_{j} X_{n}^{-1} \lambda^{-1}=\lambda q_{n j} X_{j} \lambda^{-1} \\
& =\lambda q_{n j} \phi\left(X_{j}\right) \lambda^{-1}-\lambda q_{n j} X_{n} \psi\left(X_{j}\right) \lambda^{-1}
\end{aligned}
$$


С другой стороны,

$$
\begin{aligned}
Y X_{j} Y^{-1} & =\beta\left(\phi\left(X_{j}\right)\right)-\lambda X_{n} X_{n} \psi\left(X_{j}\right) X_{n}^{-1} \lambda^{-1} \\
& =\beta\left(\phi\left(X_{j}\right)\right)-\lambda X_{n} \alpha_{n}\left(\psi\left(X_{j}\right)\right) \lambda^{-1} .
\end{aligned}
$$

Отсюда

$$
\beta\left(\phi\left(X_{j}\right)\right)=\lambda q_{n j} \phi\left(X_{j}\right) \lambda^{-1}, \quad \alpha^{-1}\left(q_{n j}\right) \psi\left(X_{j}\right)=\alpha_{n}\left(\psi\left(X_{j}\right)\right) .
$$

Заметим, что

$$
\pi(\beta(\phi))=\pi\left(\phi\left(X_{j}\right)\right)=\left(l_{1}, \ldots, l_{n}\right),
$$

поскольку $\beta$ является сопряжением с помошью $Y$. Таким образом, в $D^{*} / N$

$$
\kappa\left(\beta\left(\phi\left(X_{j}\right)\right)\right)=q_{n 1}^{l_{1}} \ldots q_{n, n-1}^{l_{n-1}} \kappa\left(\phi\left(X_{j}\right)\right)=q_{n j} \kappa\left(\phi\left(X_{j}\right)\right) .
$$

Отсюда $l_{j}=1$ и $l_{i}=0$ при $i \neq j$. Применяя аналогичные рассуждения для $\rho$, получаем, что $\phi\left(X_{j}\right)=\lambda_{j} X_{j}, \lambda_{j} \in D^{*}$. Отсюда вытекает утверждение относительно порождения $B$.

Теорема 7.2 (проблема Зарисского для лорановских многочленов). Пусть $B$ подкольцо в $\Lambda$, содержащее $D, Y$ - обратимый әлемент из $\Lambda$, причем существует такой автоморфизм $\beta$ в $B$, что

$$
Y b=\beta(b) Y \quad \text { для всех } b \in B .
$$

Предположим, что

$$
\Lambda=B\left[Y^{ \pm 1}, \beta\right] .
$$

Тогда $Y$ является одночленом от $X_{1}, \ldots, X_{r}$, причем после некоторой замень әтих переменных можно считать, что $Y=X_{1}$. B этом случае $B$ совпадает $c$ подкольцом, порохсденным

$$
D, X_{2}^{ \pm 1}, \ldots, X_{r}^{ \pm 1}, X_{r+1}, \ldots, X_{n} .
$$

ДоКАЗАТЕЛЬСтво. Обратимый элемент $Y$ в $\Lambda$ является одночленом от $X_{1}, \ldots, X_{r}$. Мультипликативная группа $\Lambda^{*}$ разлагается в полупрямое произведение:

$$
\Lambda^{*}=D^{*} \lambda\left\langle X_{1}, \ldots, X_{r}\right\rangle=B^{*} \lambda\langle Y\rangle,
$$

где

$$
\left\langle X_{1}, \ldots, X_{r}\right\rangle \simeq\left\langle X_{1}\right\rangle \times \cdots \times\left\langle X_{r}\right\rangle .
$$

Здесь $\langle\ldots\rangle$ обозначает свободную абелеву группу с указанной базой. По условию $D^{*} \subseteq$ $B^{*}$. Следовательно,

$$
\left\langle X_{1}, \ldots, X_{r}\right\rangle \simeq\left(B^{*} / D^{*}\right) \times\langle Y\rangle .
$$

Таким образом, совершая замену переменных в (46), можно считать, что $Y=X_{1}$ и $X_{2}, \ldots, X_{r} \in B^{*}$. 
Пусть $i>1$. Тогда

$$
X_{i}=\sum_{j \in \mathbb{Z}} b_{j} X_{1}^{j}, \quad b_{j} \in B
$$

Отсюда

$$
q_{1 i} X_{i}=Y X_{i} Y^{-1}=\sum_{j \in \mathbb{Z}} \alpha_{1}\left(b_{j}\right) X_{1}^{j}
$$

Следовательно, $q_{1 i} b_{j}=\alpha_{1}\left(b_{j}\right)$ для любого $j \in \mathbb{Z}$. По теореме 5.7 и (23) получаем, что $b_{j} \in D\left(X_{1} ; \alpha_{1}\right) X_{i} \cap B=D X_{i}$.

\section{§ 8. Открытые проблемы}

В заключение работы сформулируем ряд вопросов, решение которых представляется заслуживаюшим внимания.

ПроБлЕма 8.1. Пусть $F$ - тело частных обших квантовых многочленов с коэфффициентами в теле $D, n \geqslant 2$. Описать все такие пары элементов $f, g \in F^{*}$, что $f g=\lambda g \lambda^{\prime} f \lambda^{\prime \prime}$ для некоторых $\lambda, \lambda^{\prime}, \lambda^{\prime \prime} \in D^{*}$.

ПроБлЕма 8.2. Пусть $C$ - кольцо общих квантовых многочленов с телом частных $F$. Описать вложения $\phi: \Lambda \rightarrow F$. Верно ли, что при $n \geqslant 3$ сушествуют такие элементы

$$
w \in F^{*}, \quad \lambda_{1}, \ldots, \lambda_{n} \in D^{*}, \quad \varepsilon= \pm 1,
$$

что

$$
w \phi\left(X_{i}\right) w^{-1}=\lambda_{i} X_{i}{ }^{\varepsilon}, \quad i=1, \ldots, n
$$

ПробЛЕмА 8.3. Описать группу автоморфизмов $F$ над $D$.

Ответы на эти вопросы могут быть разными при $n=2$ и при $n \geqslant 3$.

ПроблЕмА 8.4. Описать правые порядки $Q$, являюшиеся правыми дробными идеалами в теле $F$. Будет ли $Q$ сопряжено в $F$ с подалгеброй из $A$ ? Описать правые порядки в $F$, содержашие $D$ и конечно порожденные над $D$. Будут ли они также сопряжены в $F$ c $A$ ?

\section{Список литературы}

1. Артамонов B. А. Проективные модули над квантовыми алгебрами полиномов // Матем. сб. 1994. Т. 185. № 7. С. 3-12.

2. Manin Yu. I. Topics in non-commutative geometry. Princeton Univ. Press, 1991. 
3. Демидов Е. Е. Квантовые группы. М.: Факториал, 1998.

4. Maltsiniotis G. Groupes quantiques et structures dirrérentielles // C. R. Acad. Sci. Paris. Sér. I. Math. 1990. V. 311. P. 831-834.

5. Alev J., Dumas F. Sur corps des fractions de certaines algébres quantique // J. Algebra. 1994. V. 170. P. 229-265.

6. Панов A.H. Тело скрученных рациональных функций и тело рациональных функций на $\mathrm{GL}_{q}(n, K) / /$ Алгебра и анализ. 1995. Т. 7. №1. С. 153-169.

7. Mc Connell J. C., Pettit J. J. Crossed products and multiplicative analogues of Weyl algebras // J. London Math. Soc. 1988. V. 38. № 1. P. 47-55.

8. Артамонов В. А. Квантовая проблема Серра // УМН. 1998. Т. 53. № 4. С. 3-77.

9. Артамонов B. A. Модули над квантовыми алгебрами полиномов // Матем. заметки. 1996. T. 59. № 4. C. $497-503$.

10. Артамонов $B$. А. Периодические модули над обшими квантовыми лорановскими полиномами // Матем. заметки. 1997. Т. 61. №1. С. 10-17.

11. Бовди А. А. Групповые кольца. Киев: УМК, 1988.

12. Artamonov V. A. Projective modules over crossed products // J. Algebra. 1995. V. 173. P. $696-714$.

13. Артамонов B.A. Автоморфизмы разложимых проективных модулей // Фундаментальная и прикладная математика. 1995. Т. 1. №1. С. 63-69.

14. McConnell J. C., Robson J. C. Noncommutative Noetherian rings. Chichester- N. Y.: John Wiley, 1987.

15. Stafford J. T. Endomorphisms of right ideals of the Weyl algebra // Trans.Amer. Math. Soc. 1987. V. 299. № 2. P. 623-639.

16. Smith P. S. An example of a ring Morita equivalent to the Weyl algebra $A_{1} / /$ J. Algebra. 1981. V. 73. № 2. P. 352-355.

17. Mikhalev A.V. Isomorphisms and anti-isomorphisms of endomorphism rings of modules // First international Tainan-Moscow algebra Workshop. Berlin - N. Y.: Walter de Gruyter, 1996. P. 69-122.

18. Quillen D. Higher algebraic K-theory // Lecture Notes Math. 1973. № 341. P. 87-147.

Поступило в редакцию 18.II. 1997 